\section{E-MRS Spring Meeting} runs the gamut of cutting-edge research

www.european-mrs.com

$\mathbf{T}$ his June, the annual European Materials Research Society (E-MRS) Spring Meeting boasted a near-record attendance of over 2,200 participants from over 60 countries, despite the current economic crisis. Convened in Strasbourg, France, and chaired by Ian W. Boyd (Melbourne Centre for Nanotechnology, Australia), Thomas K. Lippert (Paul Scherrer Institut, Switzerland), Giovanni Marletta (Università di Catania, Italy), and Rodrigo Martins (New University of Lisbon, Portugal), the meeting was organized into four symposium clusters: Biomaterials, Sensors and Surfaces; Electronic, Photonic and Optoelectronic; Carbon and Energy; and Methods and Properties. Following are a few highlights, sampling from the diverse array of innovative science and technology on display.

\section{Plenary session}

Kicking off the Plenary Session, Stuart Parkin (IBM Almaden Research Center, USA) discussed spintronics, devices based on controlling the spin of electrons. Advances in materials science have revolutionized the ability of researchers to generate, manipulate, and detect spinpolarized electrical current, said Parkin, and have facilitated this new class of spin-based sensor, memory, and logic devices. The development of spin valves and magnetic tunnel junctions provide a 1000 -fold increase in hard drive storage capacity at low cost and increased reliability. A further leap in memory capacity may come from the introduction by IBM of a novel three-dimensional-memory concept, dubbed "race track memory," which is based on the manipulation of magnetic domain walls in nanowires by a spin-polarized current. Parkin also outlined the development of a new memory concept mimicking the synaptic switching of neurons, which could lead to circuits having similar capabilities to neural networks with connecting flexibility and hence adaptability.

Continuing the theme of novel quantum circuitry, Jochen Mannhart (University of Augsburg, Germany) introduced the concept of two-dimensional electron systems at the interfaces of complex oxide systems characterized by strong electronic correlations. The origin of the interface conductivity is an electronic reconstruction caused by a built-in polar discontinuity at the interface between, for example, the insulators $\mathrm{SrTiO}_{3}$ and $\mathrm{LaAlO}_{3}$. Mannhart discussed intriguing interface properties, such as nanometer thin electron-conducting sheets, which have high charge carrier mobility at room temperature and become superconducting when cooled. At all temperatures, the carrier density reacts very sensitively to electric fields and can transition to completely insulating phases. The interfaces can be reliably patterned on a nanometer scale, enabling researchers to write quantum circuitry.

In the final plenary address, Stephen Mann (University of Bristol, UK) presented a heuristic path for constructing and transforming hybrid nanoscale objects and nanostructures. The path consisted of the synthetic construction of discrete inorganic-organic hybrid nanoobjects and higher-level nanostructured networks by self-assembly processing routes, both equilibrium and non-equilibrium. Here, self-organizing media, reaction-diffusion systems, and coupled mesophases are used to produce higher level hybrid structures under non-equilibrium conditions. As an example of a self-organizing media, Mann discussed microemulsions, which provide a confined reaction medium for the synthesis of inorganic nanoparticles and the self-assembly of those nanoparticles into organized superstructures such as linear prismatic chains. Depending

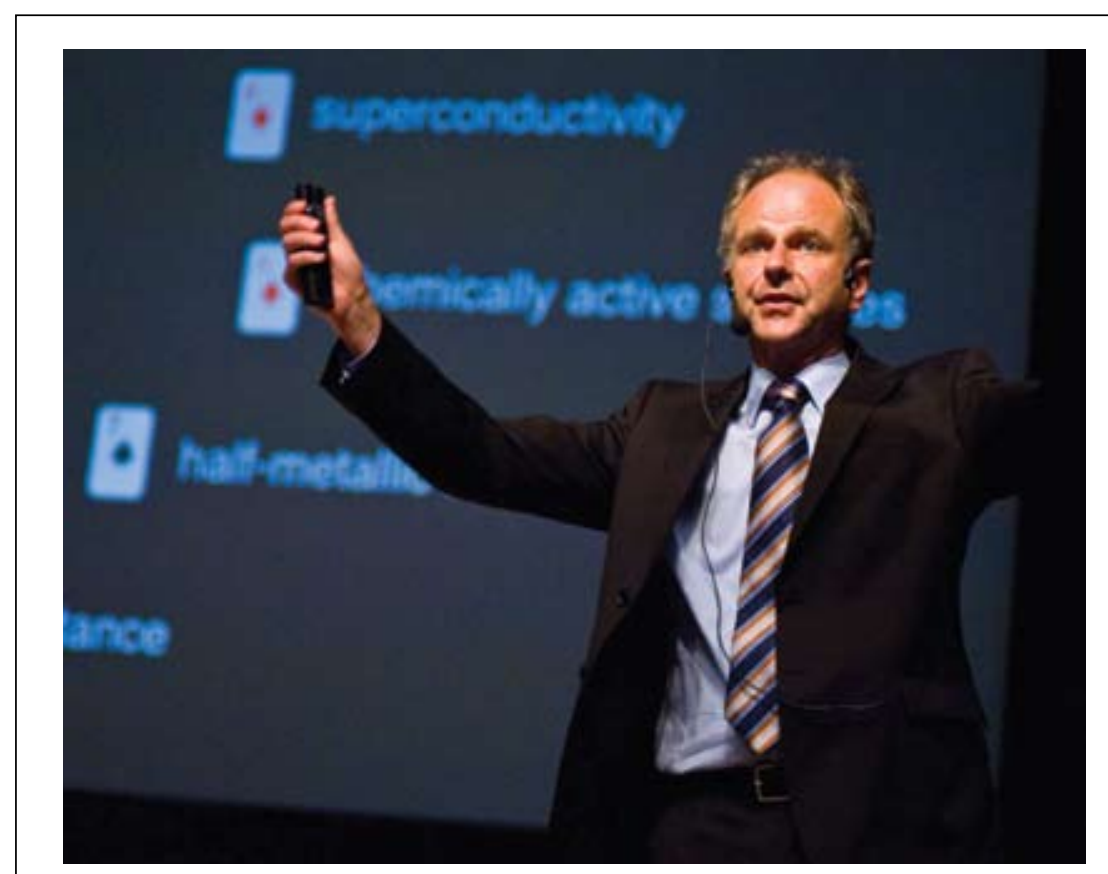

Plenary speaker Jochen Mannhart of University of Augsburg, Germany, addresses quantum circuitry at the E-MRS 2011 Spring Meeting. 


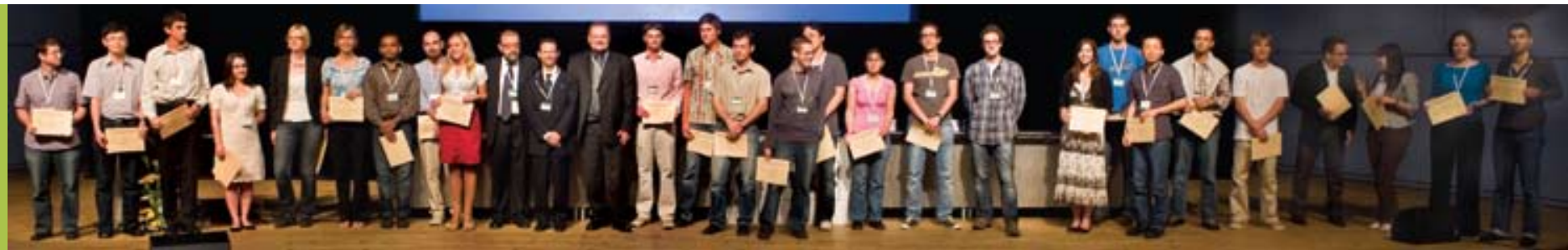

Graduate students are honored with awards at the E-MRS 2011 Spring Meeting.

on the processing route, the self-organization is highly demanding with respect to the information-generating capacity of a system, and therefore depends on a continuous flux and dissipation of energy to maintain the complex interactions between many components. These attributes are characteristic of living systems, and Mann made the comparison of static, nonequilibrium hybrid nanostructures to the dynamic, adaptive nature of biologically assembled materials.

\section{Snapshots from a few symposia \\ Biomaterials, sensors, and surfaces}

In From Embedded Sensors to Sensorial Materials, symposium co-organizer Walter Lang (University of Bremen, Germany) highlighted the move toward the integration of sensors in a variety of materials, designating it "ubiquitous sensing," a necessary counterpart to ubiquitous computing. As Lang explained it, function-scale integration, liberating the sensing element from the ballast of its substrate, narrows the size gap between technical and natural sensors. Ronald Dekker (Delft University of Technology/ Phillips Electronics, The Netherlands), in the same symposium, discussed the similar move toward "living chips," bridging microelectromechanical systems and stem cell technology. Other sessions focused on sensor integration techniques such as AerosolJet ${ }^{\mathbb{B}}$ printing, while flexible substrates received special attention based on their potential for integration, such as in textiles.

The symposium on Functional Biointerfaces addressed a growing interest in the creation, characterization, and control of interfaces between biological systems and materials. Topics covered included the challenges of creating biointerfaces and new materials from a few universal building blocks (Markus Buehler, Massachusetts Institute of Technology, USA) and innovative ways of controlling cell adhesion with conducting polymers (Hsiao-hua Yu, RIKEN Advanced Science Institute, Japan). According to Roy BarZiv (Weizmann Institute, Israel), an exciting new method to create biomolecules uses compartments within surface-bound polymer brushes, while Raphaël Lévy (University of Liverpool, UK) demonstrated that the enzyme cathepsin L degrades peptides and proteins attached to nanoparticles upon cell entry.

\section{Electronic, photonic, and optoelectronic}

A symposium on Wide Bandgap Cubic Semiconductors (WBCS) covered materials such as $3 \mathrm{C}$-SiC, diamond, carbon nitrides, and rocksalt-ZnO, establishing both their promising properties and the difficulty of their production due to low thermodynamic stability. Remarkably, each family of WBCS has a specific growth technique appropriate for stabilizing the cubic polytype, which is clearly material- and chemistry-dependent. Some promising illustrations of bulk crystals for 3C-SiC, diamond, and cubic-nitrides were presented here, but only at the laboratory scale. A major hindrance to progress is the absence of commercial sources of bulk crystalline material of large area. The need for appropriate heterosubstrates leads to the common problem of lattice and thermal-dilatation mismatches and thus to degraded crystalline quality compared to the hexagonal polytypes. It is likely that the stabilization of one of these cubic materials would assist growth of the others by providing better-adapted seeds than those currently available.

A symposium on Advanced Silicon Materials Research for Electronic and Photovoltaic Applications was dedicated to the late Ulrich Gösele of Max Planck Institute of Microstructure Physics, Germany, in honor of his prominent contributions to the field. Highlights included exciting new results in nanocrystalline and polycrystalline thin films for solar cells, point defects and their impact on device performance, progress in silicon crystal growth and improvements in measurement techniques, and a notably enthusiastic attitude to innovation.

\section{Carbon and energy}

During the Carbon- or Nitrogen-Containing Nanostructured Composite Films symposium, E-MRS President Francesco Priolo (Universita di Catania, Italy) opened the symposium and made particular reference to the work and dedication of Yves Pauleau of National Polytechnic Institute of Grenoble, France, who passed away earlier in the year. Invited speakers highlighted progress in the domain of multifunctional films consisting of nanosized particles embedded in a host matrix. Contributed presentations focused on the new developments for film production, nucleation processes, characterization, and modeling, and highlighted advances in understanding the electrical, optical, magnetic, and mechanical properties of these films. The correlations between film modeling, properties, and deposition parameters were discussed, aimed at developing new applications in thin films technologies.

The symposium Thin Film Chalcogenide Photovoltaic Materials demonstrated the dynamic community in 


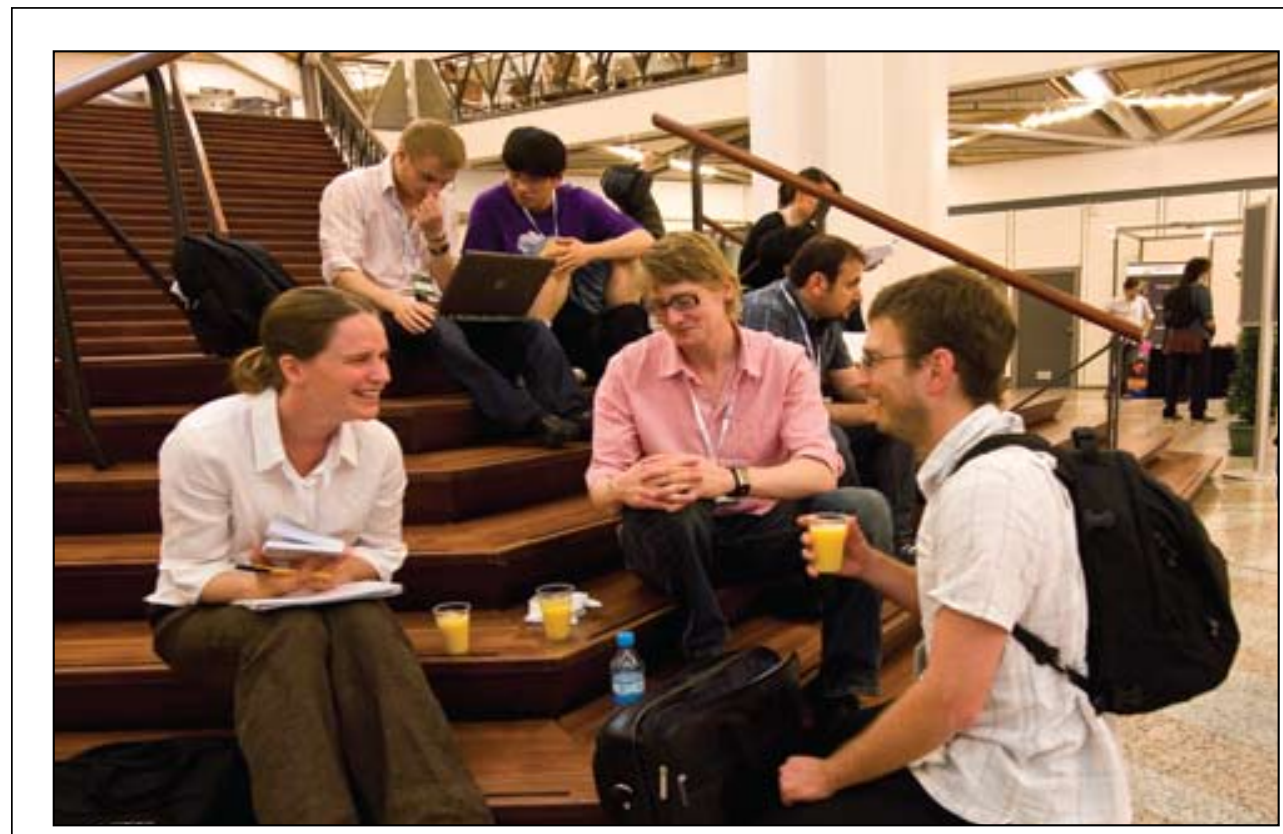

Meeting attendees converse between sessions.

as well as thermal shape memory effect. The lattice instability of Co-Ni-Ga based FSMAs has been studied for the first time by $a b$ initio calculations. The results explain the lack of nesting features on the Fermi surface and predict a different mechanism of lattice instability compared to the well-known $\mathrm{Ni}-\mathrm{Mn}-\mathrm{Ga}$ alloy system. A large high-temperature superelasticity of $8 \%$ reversible strain at $400^{\circ} \mathrm{C}$ and outstanding cyclic stability was reported in NiMn-Ga single crystals.

\section{Future meeting}

The E-MRS Spring Meeting in 2011 will be held

this field. Several records in terms of solar cell efficiency were presented: the $20 \%$ efficiency ceiling was surpassed by chalcopyrite semiconductors, $15.6 \%$ efficiency was achieved in CdTe using a low temperature process, and new kesterite compounds reached efficiencies very close to $10 \%$. Moreover, results being obtained from large-size modules are improving rapidly. The results demonstrate the levels of control being achieved with these materials in laboratories as well as at the industrial level. The symposium consolidated existing knowledge with new data on structural characterization and metastability of defects, new techniques for mapping and in situ characterization, and improved modeling of materials and devices. The symposium also made apparent the rapid development of kesterite semiconductors as a class of materials.

Solid State Ionics covered the ionic and mixed conductor materials that are key to energy conversion and storage. The increasing chemical and structural complexity in these materials has led to considerable challenges in the field, but also to exciting opportunities, as demonstrated in this symposium. Among the highlights were improved capabilities for spatially-resolved and in situ analysis of defects and elementary processes. These methods prove essential for understanding the fascinating phenomena, for example, in thin-film oxide heterostructures and redox-switching, non-volatile memory cells and devices combining ionics with electronics at nanometer length scales. Another key point was the connection between theory (ranging from ab initio calculations to phenomenological models) and experiments designed to achieve insights into the underlying mechanisms of sensors, fuel cells, and batteries, for example. A dedicated session on the occasion of Harry Tuller's 65th birthday (Massachusetts Institute of Technology, USA) with contributions from his students, colleagues, and collaborators, honored his outstanding contributions to the field.

\section{Methods and properties}

Shape Memory Materials for Smart Systems considered conventional shape memory alloys (SMAs) and ferromagnetic shape memory alloys (FSMAs) as well as considering devices and systems. A presentation on nanoscale structures of Cu-based SMAs showed that nano-pillars prepared on a single crystalline surface of $\mathrm{Cu}-\mathrm{Al}-\mathrm{Ni}$ alloy have very different thermomechanical properties than the bulk material as they show superplasticity in Nice, France on May 9-13, and will incorporate the International Union of Materials Research Societies International Conference on Advanced Materials (IUMRS ICAM) and a bilateral E-MRS/ Materials Research Society Energy conference. Further details of these events can be found on the Web site www.europeanmrs.com.

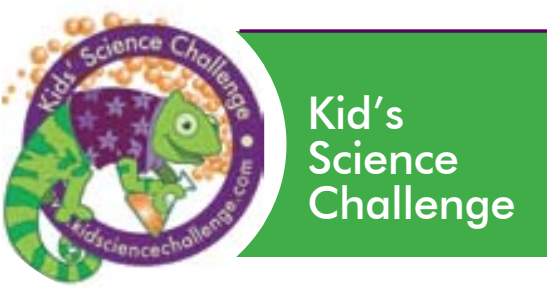

WHO Students in grades 3-6

WHAT A science competition! Kids submit ideas and experiments for scientists to solve. TOPICS-Super Stuff for Sports, Magical Microbes and Sensational Sounds

Winning students work with scientists to bring their ideas to life PLUS other fun prizes.

WHERE www.kidsciencechallenge.com

WHEN Entry deadline: February 28, 2011

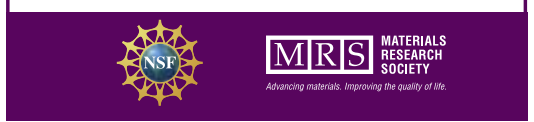


MIRS

Advancing materials. Improving the quality of life.
SECIETCH

Advancing materials. Improving the quality of life.
SECIETCH

CAMBRIDGE

UNIVERSITY PRESS

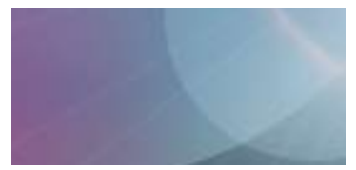

MRS publications to benefit from redesigned Cambridge Journals Online

\section{New CJO version features improved appearance, navigation, ease of use, and consistency}

$\mathrm{W}$ hen the publications of the Materials Research Society transition to Cambridge University Press in early 2011, they will be hosted on the recently re-launched Cambridge Journals Online (CJO) platform. In addition to a streamlined and updated design, the new version of CJO makes it quicker and easier to access journal content and key information, and improves the overall user experience. The key processes of content discovery, registration, and personalization, for both users and administrators, have all been simplified.

Readers of the Journal of Materials Research (JMR), MRS Bulletin, and MRS Online Proceedings Library $(O P L)$ will enjoy full advantage of CJO's robust suite of functionality, including:

- advanced search capabilitysearch across all MRS publications or narrow the focus by publication, topical area, DOI, or standard paper attributes such as title, author, volume, or issue; search parameters may also be saved for future use;

- mobile browser accessibility;

- notification of First View articles or issues as they publish, available via RSS or e-mail;

- citation export to desktop, or forward to a colleague, in formats such as EndNote, Reference Manager, ASCII Plain Text, Biblioscape, BioTex, HTMS, and Papyrus;
- reference linking-forward and backward via CrossRef, Google Scholar, and OpenURL;

- quick links to Most Read and Most Cited Articles:

- html or pdf format options for all new content, and some back content; MRS Bulletin will also be viewable in "page turning" format;

- individually tailored content alerts for complete MRS portfolioMRS Bulletin, JMR, and OPL;

- direct links to articles for authors and institutions; and

- COUNTER 3 compliant journal usage statistics for institutional subscribers.

MRS and Cambridge University Press announced their publishing partnership in June, and beginning early 2011 they will join forces to improve and expand publications for the materials research community. Watch MRS Bulletin and the MRS Web site in the coming months for developing news on MRS publications and services.

\section{CALENDAR}

Upcoming Meetings:

March 13-14, CSTIC, Endorsed

April 4-7, 17th Intl. Conf. on Microscopy of Semiconducting Materials, Endorsed April 25-29, 2011 MRS Spring Meeting, MRS

May 9-13, E-MRS 2011 Spring Meeting/IUMRS ICAM 2011; E-MRS/MRS Bilatera Conf on Energy, E-MRS, IUMRS, MRS

June 26-1, 7th Intl. Dendrimer Symp., Managed by MRS

To list an event in the Calendar, contact Robin Nazaruk, Materials Research Society, 506 Keystone Drive, Warrendale, PA 15086-7573; 724-779-2756; fax 724-779-8313; MeetingsCalendar@mrs.org.

See the September 2010 MRS BULLETIN for November 2010 Calendar entries For updates, access www.mrs.org.

- $\boldsymbol{\nabla}$ identifies a new or revised entry this month.

- "Endorsed" identifies events endorsed by MRS.

- MRS, A-MRS, B-MRS, C-MRS, E-MRS, MRS-A, MRS-I, MRS-J, MRS-K, MRS-R, MRS-S, MRS-T, M-MRS, or IUMRS at the end of an entry indicates a joint meeting or sponsorship or co-sponsorship of an event by the International Union of Materials Research Societies or one of its adhering bodies; see www.iumrs.org.

DECEMBER 2010
2-4 41st IEEE Semiconductor
Interface Specialists Conf., San Diego, CA.
Martin Frank, IBM T.J. Watson Research
Center,1101 Kitchawan Rd., Room 5-117,
Yorktown Heights, NY 10598;
914-945-1107; mfrank@ieeesisc.org;
www.ieeesisc.org.

4-8 Physical Phenomena at High Magnetic Fields (PPHMF-VII), Tallahassee, FL. Arshad Javed; 850-644-3665;

fax 850-644-5038; pphmf@fagnet.fsu.edu; http://pphmf2010.magnet.fsu.edu.

7-9 Wind Turbine Blade Manufacture 2010, Düsseldorf, Germany. Maud Lassara; 44-117- 924-9442; ml@amiplastics. com; www2.amiplastics.com/Events/ Event.aspx?code $=\mathrm{C} 371 \& \mathrm{sec}=1252$.

9-10 $\nabla$ Fuel Cells Durability and Performance, Cambridge, MA. Fuel Cells Durability 2010 Committee; 617-232-7400, ext. 211; SUBMIT@knowledgefoundation.com; www.knowledgefoundation.com.
12-15 2010 Conf. on Optoelectronic and Microelectronic Materials and Devices, Canberra, Australia. H. Hoe Tan, The Australian National University, Dept. of Electronic Materials Engineering, Canberra, ACT 0200, Australia; 61-2-6125 0356; hoe.tan@anu.edu.au; http://commad2010.anu.edu.au.

\section{JANUARY 2011}

5-7 2nd Conf. on Advances in Microfluidics and Nanofluidics and Asian-Pacific Intl. Symp. on Lab on Chip (AMN-APLOC 2011), Singapore. amnaploc11@ntu.edu. sg: www.amnaploc2011.org.

9-13 2nd Intl. Congress on Sustainability Science and Engineering (ICOSSE'11), Tucson, AZ. Glenn Schrader, University of Arizona, 1209 East 2nd St., Tucson, AZ 85721-0072; 520-621-6596; schrader@ arizona.edu; www.icosse11.org.

17-19 The Coatings Summit 2011 Washington, $D C$. Friederike Plasswich, Vincentz Network, Plathnerstr. 4c, Hannover, 30175, Germany; 49-511-9910279; fax 49-511-9910-099; friederike. plasswich@vincentz.net; www.europeancoatings.com/events/microsites/summit. 
20-21 2011 Intl. Workshop on Dielectric Thin Films for Future Electron Devices, Tokyo, Japan. ml-iwdtf@hiroshima-u.ac.jp; http://home.hiroshima-u.ac.jp/iwdtf.

23-27 24th Intl. Conf. on Micro Electro Mechanical Systems, Cancun, Mexico. MEMS 2011 Conf., c/o Preferred Meeting Management, Inc., 307 Laurel St., San Diego, CA 92101-1630; 619-232-9499; fax 619-232-0799; info@ieee-mems2011.org; www.ieee-mems2011.org.

\section{FEBRUARY 2011}

7-11 $\nabla$ 5th Intl. Conf. on Advanced Materials and Nanotechnology, Wellington, New Zealand. Conf. Secretariat, PO Box 24 078, Mail Centre Manners St., Wellington, New Zealand; 64-4-384-1511; amn-5@confer. co.nz; www.confer.co.nz/amn-5.

16-18 Intl. Nanotechnology Exhibition and Conf., Tokyo, Japan. Secretariat of nano tech executive committee, Chiyoda Bldg.1-5-18, Sarugakucho, Chiyoda-ku, Tokyo, 101-8449, Japan; 81-3-3219-3567; fax 81-3-3219-3628; nanotech@ics-inc. co.jp; www.nanotechexpo.jp/en.

27-3 TMS 2011 Annual Meeting and Exhibition, San Diego, CA. TMS Meeting Services,184 Thorn Hill Rd., Warrendale, PA 15086-7514; 724-776-9000, ext. 243; fax 724-776-3770; mtgserv@tms.org; www.tms.org/meetings/annual-11/ AM11home.aspx.

\section{MARCH 201}

13-14 China Semiconductor Technology Intl. Conf. (CSTIC), Shanghai, China. April Peng, SEMI China, 18G/H, Tower 2, Hua Ao Ctr., 31 Zizhuyuan Rd., Beijing, 100089, China; 86-10-519060-86;

fax 86-10-51906087; apeng@semi.org; www.semi.org.cn/cstic. Endorsed.

14-17 2011 Intl. Laser Safety Conf. San Jose, CA. Laser Institute of America's Conf. Dept.; 800-34-LASER; ilsc@ laserinstitute.org; www.laserinstitute. org/ilsc.

14-17 Navigating Chemical Compound Space for Materials and Bio Design, Los Angeles, CA. http://e-math.ams.org/ meetings/calendar/2011_mar14-jun17 losangeles.htm

21-25 American Physical Society (APS) Physics - March Meeting, Dallas, TX. American Physical Society, One Physics Ellipse, College Park, MD. 20740-3844; 301-209-3200; fax 301-209-0865 www.aps.org/meetings/march/index.cfm.

$22 \nabla$ CS Europe - Compound Semiconductor Conf., Frankfurt, Germany. CS Europe; info@cseurope.net; www. cseurope.net.

27-31 $\boldsymbol{\nabla}$ 241st ACS National Meeting Anaheim, CA. ACS; 202-776-8044; nationalmeetings@acs.org; www.acs.org/ anaheim2011.
29-31 European Coatings Show 2011, Nuremberg, Germany. Nicole Steinbach, Vincentz Network GmbH and Co. KG, Plathnerstr. 4c, Hannover, 30175 Germany; 49-5-11-99-10-274; fax 49-511-99-10-279; nicole.steinbach@vincentz. net; www.european-coatings-show.com/ en/press/ars12/ars25/?arspageid=25. pm.6354.

30-31 $\nabla$ World Materials Perspectives (WMP) 2011, Nancy, France. info@wmproadmaps.org; www.wmp-roadmaps.org.

\section{APRIL 2011}

4-7 17th Intl. Conf. on Microscopy of Semiconducting Materials, Cambridge UK. Claire Garland, Institute of Physics, 76 Portland PI., London, W1B 1NT, UK; 44-20-7470-4800; fax 44-20-7637-4266 claire.garland@iop.org; www.msm2011. org. Endorsed.

5-6 NanoManufacturing Conf. and Exhibits, Oakbrook Terrace, IL. Society of Manufacturing Engineers: 800-733-4763; service@sme.org; www.sme.org/nanomanufacturing.

16-21 2011 Society of Vacuum Coaters (SVC) Technical Conf., Chicago, IL. SVC, 71 Pinon Hill PI. NE, Albuquerque, NM 87122-1914; 505-856-7188; fax 505-856-6716; svcinfo@svc.org www.svc.org

18-19 $\nabla$ 12th Annual IEEE Wireless and Microwave Technology (WAMI) Conf., Clearwater, FL. www.wamicon.org.

25-29 2011 MRS Spring Meeting, San Francisco, CA. Materials Research Society, 506 Keystone Dr., Warrendale, PA 15086, USA; 724-779-3003; fax 724-779-8313; info@mrs.org; www.mrs.org. MRS

\section{MAY 2011}

2-6 International Conference on Metallurgical Coatings and Thin Films (ICMCTF 2011), San Diego, CA. Mary Gray; 703-266-3287; icmctf@mindspring.com. www.avs.org/conferences/ICMCTF.

9-13 E-MRS 2011 Spring Meeting/ IUMRS ICAM 2011, including E-MRS/ MRS Bilateral Conf. on Energy, Nice, France. European Materials Research Society; 33-3-88-10-63-72; fax 33-3-8810-62-93; emrs@emrs-strasbourg.com; wWW. emrs-strasbourg.com/ICAM2011. E-MRS, IUMRS, MRS

15-18 The ConFab, Las Vegas, NV. Jo-Ann Pellegrini; 650-946-3169; joannp@ pennwell.com; www.theconfab.com

20-25 XXVI Congress of the Intl. Society for the Advancement of Cytometry, Baltimore, MD. Roya Jaseb, 9650 Rockville Pike, Bethesda, MD 20814; 301-634-7017 fax 301-634-7014; isac@faseb.org; www. isac-net.org.

22-27 Society for Information Display (SID) 2011, Los Angeles, CA. $1475 \mathrm{~S}$. Bascom Ave., Ste. 114, Campbell, CA 95008; 408-879-3901; fax 408-879-3833; office@sid.org; www.sid.org
23-26 $\nabla$ Advanced Aerospace Materials and Processes Conf. and Exposition (AeroMat) 2011, Long Beach, CA. ASM Intl., Member Service Center, 9639 Kinsman Rd., Materials Park, $\mathrm{OH} 44073-0002$ USA; 800-336-5152; fax 440-338-4634; customerservice@asminternational.org; www.asminternational.org/content/Events/ aeromat.

\section{JUNE 201}

5-9 16th Intl. Conf. on Solid- State Sensors, Actuators and Microsystems (Transducers '11), Beijing, China. China Intl. Conf. Center for Science and Tech, Rm. 60742, Unit 7, Suyuan Apt., Beijing Friendship Hotel No.1, Zhongguanchuan Nandajie, Beiijing, 100873, China; fax 8610-62174126; info@transducers11-beijing. org; http://transducers11-beijing.org.

17-20 Glass Performance Days Finland, Tampere, Finland. Jorma Vitkala, Glaston Finland/GPD, Vehmaistenkatu 5, Tampere, 33730, Finland; 358-10-500-6216; Jorma. Vitkala@gpd.fi; http://gpd.fi.

19-25 2th World Conf. on Titanium (Ti-2011), Beijing, China. Hongkang Zhu, Ti-2011 Secretariat, No. 96, Weiyang Rd., Xi'an, Shaanxi, 710016, China; 86-2986266570; fax 86-29-86231101; sec@ ti-2011. com; www.ti-2011.com.

26-1 7th Intl. Dendrimer Symp. Gaithersburg, MD. Materials Research Society; 724-779-3003; fax 724-7798313; info@mrs.org; www.mrs.org. Managed by MRS

26-1 Intl. Conf. on Materials for Advanced Technologies (ICMAT2011) Suntec, Singapore. ICMAT2011 Secretaria Office, Meeting Matters Intl., 25 Hindoo Rd., Singapore, 209116; 65-6341-7229; fax 65-6341-7269; icmat2011@meetmatt. net; www.mrs.org.sg/icmat2011.

\section{JULY 2011}

18-22 7th Intl. Congress on Industrial and Applied Mathematics (ICIAM 2011) Vancouver, BC, Canada. info@iciam2011. com; www.iciam2011.com.

\section{AUGUST 2011}

\section{4-19 Intl. Materials Research} Congress (IMRC) 2011, Cancun, Mexico. Info@mrs-mexico.org.mx www.mrsmexico.org.mx/imrc2010; www.mrs.org/ IMRC_2011. M-MRS, MRS

22-26 24th Intl. Conf. on Amorphous and Nanocrystalline Semiconductors (ICANS 24), Nara, Japan. www.icans24.org/index. html.

\section{SEPTEMBER 2011}

11-16 Intl Conf. on Silicon Carbide and Related Materials (ICSCRM) 2011, Cleveland, $\mathrm{OH}$. Barbara Kakiris, NASA Glenn Conf. Liaison, 21000 Brookpark Rd., MS 3-5, Cleveland, OH 44135; 216-433-2513; fax 216-977-7133; Barbara.L.Kakiris@ nasa.gov; www.icscrm2011.org.

\section{OCTOBER 201}

2-6 Federation of Analytical Chemistry and Spectroscopy Societies 2011 (FACSS 2011), Reno, NV. Federation of Analytical Chemistry and Spectroscopy, PO Box 24379, Santa Fe, NM 87502, USA; 505-820-1648; facss@facss.org; http://facss.org.

2-7 4th Intl. Conf. on Electrophoretic Deposition: Fundamentals and Applications (EPD 2011), Puerto Vallarta, Mexico. Engineering Confs. Intl., 32 Broadway, Ste. 314, New York, NY 10004; 212-514-6760; info@engconfintl.org; www.engconfintl. org/11ab.html.

\section{2-7 XXXV Intl. Scientific Basis} for Nuclear Waste Management Symp. Buenos Aires, Argentina. Ricardo Carranza, Comisión Nacional de Energía Atómica; 54-11-6772-7488; fax 54-116772-7362; carranza@cnea.gov.ar; www.cnea.gov.ar/mrs2011. Endorsed

12-14 Intl. Congress and Exhibition Euro PM2011, Barcelona, Spain. European Powder Metallurgy Association, Talbot House, Market St., Shrewsbury, SY1 1LG, UK; 44-1743-248899; info@epma.com; www.epma.com/pm2011.

16-20 Materials Science and Technology 2011 Conf. and Exhibition (MS\&T'11), Columbus, $\mathrm{OH}$. Meeting Services, TMS 184 Thorn Hill Rd., Warrendale, PA 15086 724-776-9000, ext. 243; mtgserv@tms.org.

31-2 26th Materials Information Society (ASM) Heat Treating Society Conf. and Exposition, Cincinnati, OH. ASM Member Service Center, 9639 Kinsman Rd. Materials Park, $\mathrm{OH}$ 44073-0002. 440-338-5151, ext. 0; fax 440-338-4634 memberservicecenter@asminternational. org; http://hts.asminternational.org/ content/Events/Heattreat/index.jsp.

\section{NOVEMBER 201}

27-1 Fray Intl. Symp., Cancun, Mexico Florian Kongoli, Flogen Technologies Inc. 1255 Laird Blvd., Ste. 388, Mont-Royal, QC, H3P2T1, Canada; 514-344-8786 ext. 220; fax 514-344-0361; fkongoli@flogen. com; www.flogen.com/FraySymposium.

28-2 2011 MRS Fall Meeting, Boston, MA. Materials Research Society, 506 Keystone Dr., Warrendale, PA 15086, USA; 724-779-3003; fax 724-779-8313; info@ mrs.org; www.mrs.org/F11. MRS.

\section{DECEMBER 2011}

11-15 2nd Nano Today Conf., Waikoloa Beach, HI. 2nd Nano Today Conf. Secretariat, Elsevier, The Boulevard, Langford Ln., Kidlington, Oxford, OX5 1GB, UK; 44-1865-843051; nanotoday2011@ elsevier.com; www.nanotoday-conference. com. 


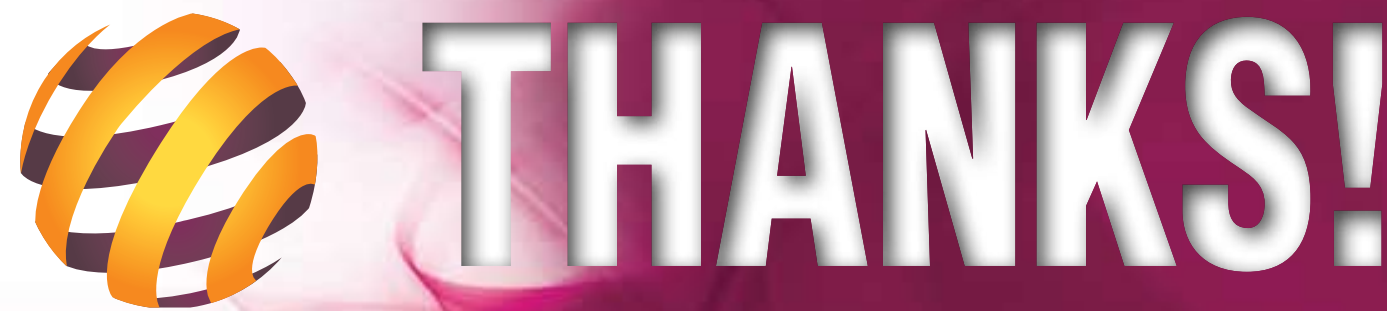

The following events at the 2010 MRS Fall Meeting have been funded, in part, by the generous contributions of these organizations.

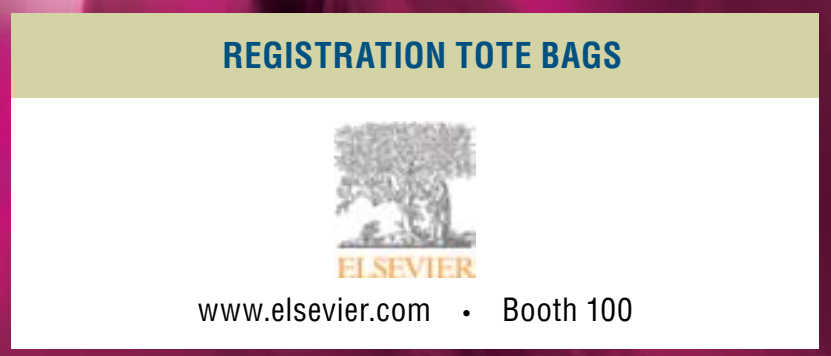

POSTER AWARDS
$\therefore \because \because$ EEI ${ }^{*}$
$\because \because \because$ E
www.fei.com Booth 617

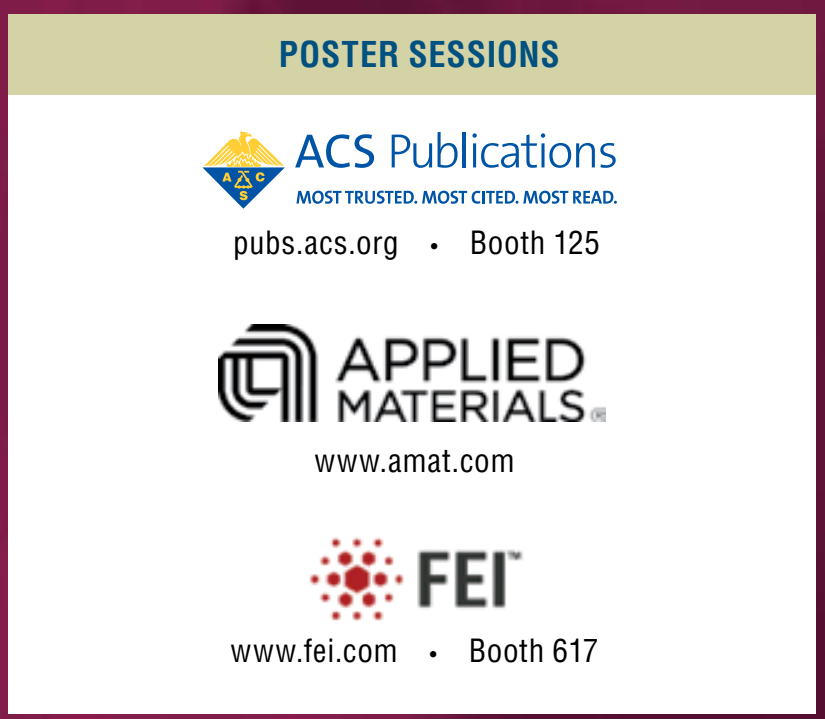

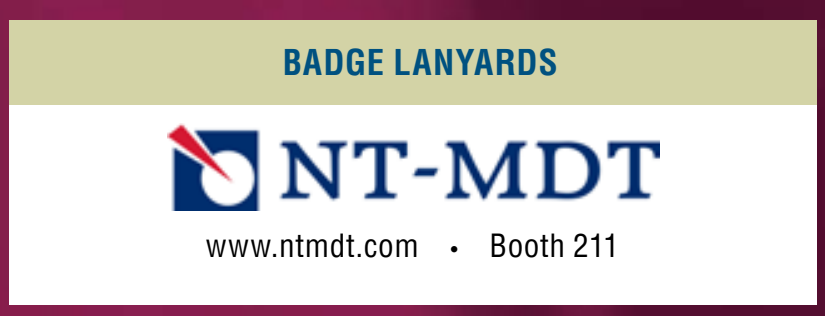

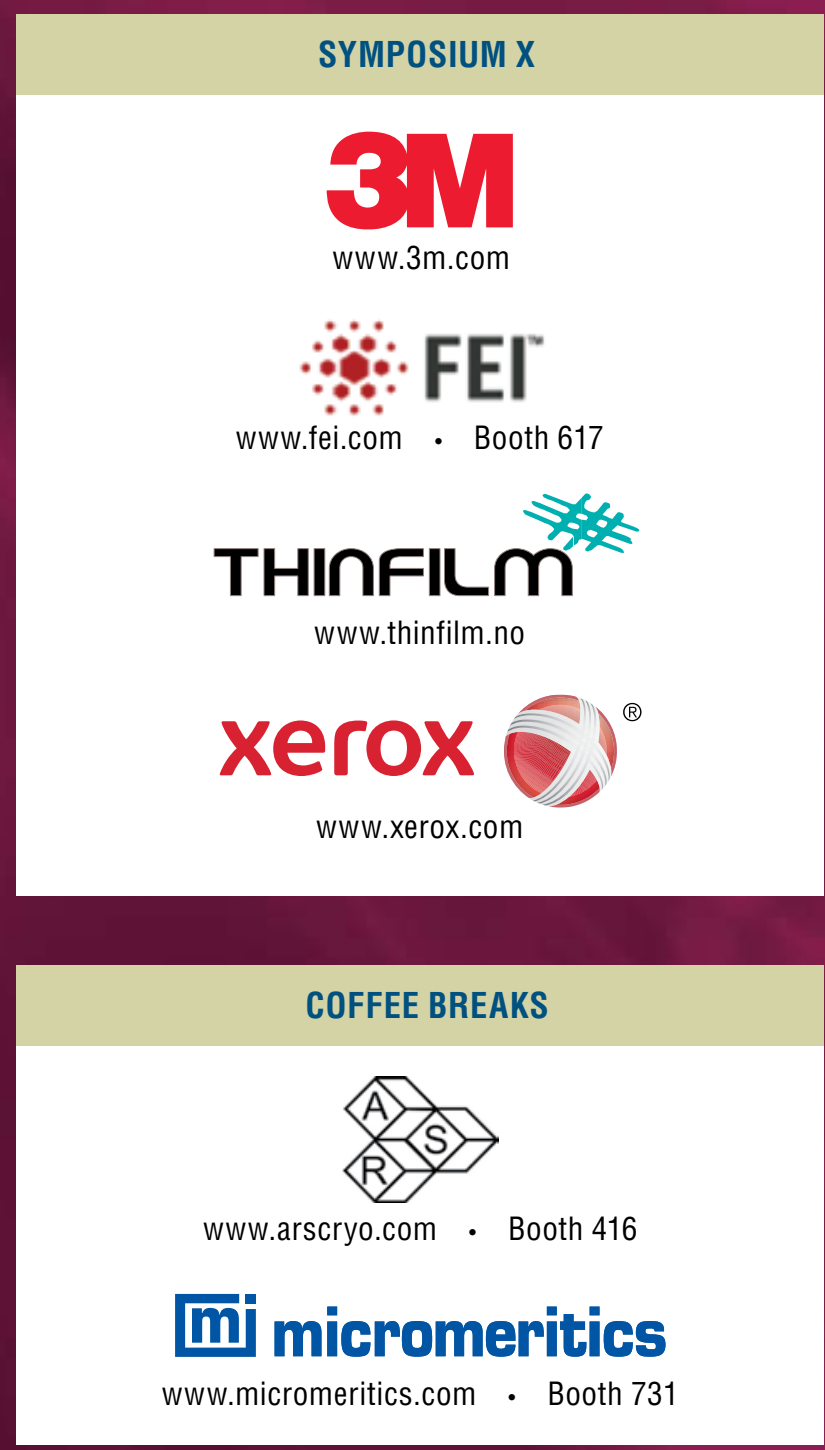

WOMEN IN MATERIALS SCIENCE BREAKFAST

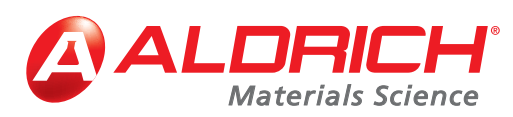

www.sigma-aldrich.com/matsci • Booth 1025 


\section{MIIISFALL J EXHIBTOOS \\ Hynes Convention Center \\ Level 2 \\ Tuesday, November 30 \\ 11:00 am - 5:30 pm \\ Wednesday, December 1 11:00 am - 6:00 pm \\ Thursday, December 2 \\ 10:00 am - 1:30 pm}

\begin{abstract}
A \& N Corporation
wWw.ancorp.com

Vacuum Chambers; Vacuum Components;

Vacuum Measurement

\section{Across International LLC}

Booth 1204

www.acrossinternational.com

Planetary Ball Mills; Vacuum Drying Ovens;

Pellet Press

\section{ACS Publications}

pubs.acs.org

Journals (Web and Print); Magazines; Books

\section{Advanced Diamond}

Technologies, Inc.

www.thindiamond.com

NaDiaProbes (All-diamond AFM Probes); UNCD

Diamond Wafers; UNCD Components
\end{abstract}

Advanced Polymer Materials Inc. Booth 822 www.apmpolymers.com

Biodegradable Polymers; Block Copolymers;

Functional Polymers

Advanced Research Systems, Inc. Booth 416 www.arscryo.com

Closed and Open Cycle Cryogenic Systems;

Cryogenic Probe Station

Aep Technology, Inc.

www.aeptechnology.com

Dual Mode Profiler; Optical/Contact Stylus Profiler:

Film Thickness/Stress Monitor

Agilent Technologies Booth $\mathbf{4 0 1}$
www.agilent.com/find/nano
Atomic Force Microscopes; Nanoindentation
Systems; Nanomechanical Testing Systems;
Field Emission Scanning Electron Microscopes;
Microwave Network Analyzers

\begin{tabular}{ll}
\hline AIST-NT, Inc. & Booth 1307
\end{tabular}

www.aist-nt.com

Atomic Force Microscopes; AFM-Raman;

SPM Accessories

aixACCT Systems GmbH

Booth 1033

WWW.aixacct.com

Materials Analyzers; aixDBLI

\section{AIXTRON AG \\ Booth 425 \\ WWW.aixtron.com \\ MOCVD and OVPD Equipment; ALD, AVD and}

CVD Equipment; PECVD Equipment

AJA International, Inc.

Booth 313

www.ajaint.com

Sputtering Systems; Sputter Sources

Sputter Targets

\section{Aldrich Materials Science}

Booth 1025

www.sigma-aldrich.com/matsc

Organic Electronics; Alternative Energy;

Nanomaterials; Polymerization Tools; Materials for Thin Films

\section{Alfa Aesar, a Johnson}

Booth 925

Matthey Company

www.alfa.com

High-Purity Metals; Evaporation Materials; Ceramics

\section{American Institute of Physics}

Booth 117

www.aip.org

Physics Journals; Online Hosting; Conference Proceedings

\section{American Physical Society}

Booth 119

publish.aps.org

Physics Journals; Online Journals

Anasys Instruments Corp.

Booth 1006

www anasysinstruments com

Nanoscale Infrared Spectroscopy (nanolR);

Nanoscale Thermal Analysis (nano-TA)

VESTA Local Thermal Analysis System

Andeen-Hagerling, Inc.

Booth 521

www.andeen-hagerling.com

Ultra-precise Capacitance Bridges; Ultra-stable Reference Capacitors

\section{Anfatec Instruments AG}

Booth 1211

www.anfatec.de

AFM and STM; LockIn Amplifiers; SPM Controllers

Angstrom Engineering Inc.

Booth 1019

www.angstromengineering.com

Sputter Deposition System; Thermal Evaporation

System; Electron Beam Deposition System

\section{Angstrom Sciences}

Booth 1306

www.angstromsciences.com

Magnetrons; Sputtering Material; Sputtering Cathodes

Angstrom Scientific Inc.

Booth 708

WwW.angstrom.us

Nano and Micro Manipulators; TM3000 Benchtop

SEM; Used Electron Microscopes and FIBs

Angstrom Sun Technologies, Inc. Booth 929 www.angstec.com

Spectroscopic Ellipsometer;

Microspectrophotometer; Thin Film Thickness

Measurement Tools; Reflectometer

\section{Annealsys}

www.annealsys.com

RTP; RTCVD; Annealing; MOCVD; Spray-CVD

\section{LPCVD}

Anter Corporation

www.anter.com

Laser Flash Systems; Thermal Conductivity Meters;

Dilatometers

\section{Anton Paar USA}

Booth 908

www.anton-paar.com

X-Ray Scattering Instrumentation

Applied Nanostructures, Inc.

Booth 828

www.appnano.com

AFM Probes; SPM Probes; MEMS

Applied Surface Technologies

www.co2clean.com

CO Snow Jet; Surface Cleaning; AFM Sample

\section{Cleaning}

Asylum Research

WwW.AsylumResearch.com

Cypher AFM; MFP-3D AFM; Atomic Force/Scanning

Probe Microscopes

\section{attocube systems $A G$}

Booth 507

www.attocube.com

Nanopositioning Systems; Microscopes;

Interferometers

B\&W Tek, Inc.

www.bwtek.com

Confocal Raman Spectrometers; UV/VIS/NIR

Spectrometers; Portable Raman Spectrometers

Baden-Württemberg International Booth 111

www.bw-i.de

Discover Excellence-Made in Germany
Biolin Scientific, Inc.

www.biolinscientific.com

Quartz Crystal Microbalance with Dissipation

Monitoring; Farfield Dual Polarization Interferometer; Attension Theta Optical Tensiometer

\section{Biologic USA}

Booth 805

www.bio-logic.us

Electrochemistry; Corrosion; Battery and Fuel Cell Testing

Blue Wave Semiconductors, Inc. Booth 806

www.bluewavesemi.com

Substrate Heaters; Thin Film Coatings; PVD, CVD Deposition Systems

Brooks Automation, Inc.

Booth 1305

www.brooks.com

CTI Cryogenics Cryopumps; Granville-Phillips

Vacuum Measurement Solutions; Polycold Systems Cryogenic Refrigeration

\section{Bruker}

Booth 301

www.bruker.com

X-Ray Diffraction \& Fluorescence Instruments;

Raman Spectroscopy; Atomic Force Microscopy

\section{Bruker Nano Surfaces Business}

(formerly Veeco Instruments Inc.)

Booth $\mathbf{3 0 0}$

www.bruker.com

Atomic Force Microscopes; Optical Interferometric

Profilers; Stylus Profilers; Confocal Microscopes

Cambridge Nanotech Inc.

www cambridgenanotech.com

Booth 622

ALD Systems: Savannah; Fiji; Phoenix; Tahiti

Cambridge University Press

Booth 107

www.cambridge.org/us

Books; Journals

Capovani Brothers Inc.

www.capovani.com

Used Scientific Equipment

Carl Zeiss Microlmaging, LLC

www.zeiss.com/materials

Microscopes; Digital Imaging Systems

Carl Zeiss NTS, LLC

www.zeiss.com/nts

Scanning Electron Microscopy; Helium Ion

Microscopy; Transmission Electron Microscopy

CEM Corporation

Booth 706

www.cem.com

Discover ${ }^{\circledR}$ Microwave Synthesizers; MARS ${ }^{\mathrm{TM}}$

Microwave Synthesizers; Phoenix ${ }^{\circledR}$ Microwave

Ashing \& Muffle Furnace

\section{CETR}

Booth 724

www.cetr.com

Universal Nano+Micro+Macro Materials Testers

Chemat Technology, Inc.

Booth 201

www.chemat.com

Spin Coater; Coating Equipment; Chemical

Precursors

\section{Clarkson University, Materials}

Booth 1301

Science \& Engineering

www.clarkson.edu

ColdEdge Technologies, Inc.

www.coldedgetech.com

Cryostats; Cryocoolers; Cryogenics
Booth 821 
COMSOL, Inc.

Booth 1021

WWW.comsol.com

COMSOL Multiphysics; COMSOL Reaction

Engineering Lab

Cryo Industries of America, Inc. Booth 910

WWW.cryoindustries.com

Continuous Flow \& Reservoir Cryostats; MFRM-

Nano MRI; 4K and 10K Mechanical Closed-Cycle

Refrigerators

CrystalMaker Software Ltd. $\quad$ Booth $\mathbf{9 3 1}$
www.crystalmaker.com
CrystalMaker; CrystalDiffract; SingleCrystal

CSM Instruments Inc. Booth 517

www.csm-instruments.com

Instrumented Indentation; Scratch Testers;

Tribometer Pin-on-Disk; Calotest; AFM \& Conscan

Imaging

DCA Instruments, Inc.

Booth 522

www.dca.fi

UHV Deposition Systems; MBE Components;

PVD Systems

\section{Delong America Inc.}

Booth 222

www.lv-em.com

Benchtop Combined TEM and SEM; Low Voltage TEM

Denton Vacuum, LLC

Booth 913

www.dentonvacuum.com

Sample Preparation Tools; High Vacuum Carbon

Evaporators; Thin Film Deposition Systems

Digital Surf SARL

Booth 203

www.digitalsurf.com

Surface Imaging and Analysis Software for SPMs:

Microscopes; Profilometers.

DTF Technology GmbH Booth 1022

www.dtf-technology.de

Flash Lamp Annealing Systems; ALD Tools:

Vacuum \& Deposition Equipment

Duniway Stockroom Corporation Booth 623

www.duniway.com

Vacuum Supplies; Vacuum Hardware; Ion Pumps

\section{Ebatco}

Booth 221

www.ebatco.com

Lab Services; Materials Consulting; Scientific Instruments

Ecopia Corp.

Booth 604

www.ecopia21.co.k

Variable Temperature Hall Effect Measurement

System; Probe Station; Heat Treatment System

eDAQ, Inc.

Booth 620

www.edaq.com

Electrochemical Impedance Spectroscopy;

Electrochemistry Systems; Data Recorders

EDAX Inc.

Booth 916

www.edax.com

Energy Dispersive X-ray; EBSD; X-ray Detectors

\section{Electron Microscopy}

Booth 606

Sciences/Diatome

www.emsdiasum.com

Microscopy Supplies; Material Specimen Preparation

Elsevier

Booth 100

www elsevier.com

Books; Journals; Electronic Products

Evans Analytical Group (EAG) Booth 812

www.eaglabs.com

Analytical Services

$\begin{array}{ll}\text { FEI Company } & \text { Booth } \mathbf{6 1 7} \\ \text { Www.fei.com } & \\ \text { SEM; TEM; DualBeam SEM/FIB } & \end{array}$

SEM; TEM; DualBeam SEM/FIB

FibeRio Technology Corporation

Booth 921

www fiberiotech com

Nanofiber Processing Equipmen
First Nano, A Division of CVD

Booth 919

Equipment Corporation

www. firstnano.com

Turn-Key CVD Process/Gas Delivery/Exhaust Gas

Treatment Equipment

Fischione Instruments

Booth 309

www.fischione.com

Electron Microscope Accessories; Plasma Cleaner; Ion Mill

FlackTek, Inc.

Booth 733

www.speedmixer.com

Mixing Machines; Laboratory Mixers;

High-speed Mixing

FUJIFILM Dimatix, Inc.

Booth 1007

www.dimatix.com

Materials Deposition Printer; Materials Deposition

Cartridge; Other Printheads and Systems

Gatan, Inc.

Booth 601

www.gatan.com

Nanomaterial, Photovoltaic Material and Opto-

electronic Material Characterization Tools for EM

Geib Refining Corp.

Booth 906

www.geibrefining.com

Reclaim of Precious Metals; Gold Refining;

Shield Cleaning

Gelest Inc.

Booth 1035

www.gelest.com

Silanes; Silicone; Metal Organics

Goodfellow Corporation

www.goodfellowusa.com

Metals and Materials for Research \& Development

Hamamatsu Corporation

Booth 525

www.sales.hamamatsu.com

Quantum Yield Measurement; NIR; VUV;

Electron Detection and Imaging

\section{HeatWave Labs Inc.}

Booth 624

www cathode com

Substrate Heaters; Dispenser Cathodes;

Electron Guns

\section{Herzan LLC}

Booth 608

www.herzan.com

Vibration Isolation; Acoustic Enclosures:

Site Survey Kit

Hielscher USA, Inc.

www. hielscher.com

Ultrasonic Mixers; Dispersion Technology;

Sonochemistry

\section{HORIBA Scientific}

www.horiba.com/scientific

Thin Film; Raman; Ellipsometry

Hummingbird Scientific LLC

www.hummingbirdscientific.com

TEM Specimen Holders

Huntington Mechanical

Laboratories, Inc.

www.huntvac.com

Vacuum Chambers; Vacuum Feedthroughs;

vacuum Hardware

Hysitron, Inc.

www hysitron com

PI 95 TEM Picolndenter; PI 85 SEM Picolndenter

Hysitron, Inc.

www.hysitron.com

In-situ Nanomechanical Test Instruments: TI 950

Tribolndenter $^{\circledR}$; TI 750 Ubi $^{\text {TM}}$; TS 75 TriboScope $^{\circledR}$

Image Metrology A/S

Booth 912

www.imagemet.com

SPIP Image Analysis Software

Imina Technologies

Booth 722

www.imina.ch

Nano Manipulators; Nano Electrical

Probing Systems
Inel, Inc.

www.inel.fr

X-ray Diffractometers; Position Sensitive Detectors;

Power Diffractometers

INFICON

Booth 817

Www.inficon.com

Thin Film Deposition; Quartz Crystal Microbalance

Research; Vacuum Gauges and Components

Innovative Technology, Inc.

Booth 730

www gloveboxes com

Glove Boxes; Vapor Deposition Equipment

Spin Coaters; Gas Purification Systems

\section{International Centre for}

Diffraction Data (ICDD)

www.icdd.com

Powder Diffraction File (PDF-4+; PDF-4/Organics;

PDF-2; PDF-4/Minerals)

International Union of

Booth 124

Crystallography

www.iucr.org

Journals; Reference Books; Online Services

Intlvac

www.intlvac.com

Booth 830

Ion Beam Etch Systems; Physical Vapor Deposition

IOP Publishing

publishing.iop.org

Booth 108

Leading journals, including Nanotechnology, Journal of Physics D: Applied Physics and MSMSE

J microTechnology, Inc.

www.jmicrotechnology.com

Booth 918

Probing Equipment; Probes; Optical Systems

Janis Research Company, Inc.

Booth 408

www.janis.com

Micromanipulated Probe Stations; $4 \mathrm{~K}$ and $10 \mathrm{~K}$

Mechanical Closed-Cycle Refrigerators; Continuous

Flow and Reservoir Cryostats

Japan Science and

Technology Agency

www.jst.go.jp/tt/EN/cips_details.htm

Technology Licensing for Categories including New

Materials, Electronic Devices and Water Cleaning

Japan Society of Applied Physics Booth 110

www.jsap.or.jp/english/index.html

Journals, such as Applied Physics Express, Japanese Journal of Applied Physics

JASCO

WWW.jascoinc.com

Spectroscopy Instrumentation; Chromatography

Instrumentation; Raman; Nanomaterials

Characterization; FT-IR Microscopy

JEOL USA, Inc.

Booth 600

WWW.jeolusa.com

TEM; SEM; NMR

Kaufman \& Robinson, Inc.

www.ionsources.com

Gridded Ion Sources; Griddless Ion Sources;

Plasma Sources

Keithley Instruments, Inc.

www.keithley.com

Complete Delta Mode System; Electrometer/High

Resistance Meter; SourceMeter Instrument

Keyence Corporation

Booth 823

www.keyence.com

VHX-1000 Digital Microscope; VK-9700 3D Lase

Scanning Microscope

Kimball Physics, Inc.

www.kimballphysics.com

Booth 700

UHV Electron and Ion Guns; Multi-CF Chambers and

Fittings; Cathodes/Detectors/eV Parts

KLA-Tencor Corporation

Booth 701

www.kla-tencor.com

Stylus Profiler; Universal Microscope 
KP Technology Ltd.

www.kelvinprobe.com

Scanning Kelvin Probe; Surface Photovoltage

Kelvin Probe; UHV Kelvin Probe

\section{Kurt J. Lesker Company}

Booth 411

www.lesker.com

Pure Targets and Materials; Vacuum Components;

Deposition Systems; Target Bonding Services;

UHV Manipulation

\section{Kyoto Environmental \\ Booth 1303 \\ Nanotechnology Cluster \\ www.kyo-nano.jp/index.php?lang=en \\ Electricity-saving Devices; Catalyst Nanotechnology \\ for New Energy; Functional Metals; UV Sensor; Infrared Sensor}

Lake Shore Cryotronics, Inc.

Booth 800

www.lakeshore.com

Hall Effect Measurement Systems; Probe Stations;

Cryogenic Instruments and Sensors

Laser Quantum Ltd.

www.laserquantum.com

Lasers for Raman; OPSS Lasers; Lasers for

Fluorescence

\begin{tabular}{lc}
\hline Leica Microsystems Inc. & Booth 316 \\
www.leica-microsystems.com & \\
Leica EM TXP Planer; EM TIC020 Ion Beam Cutter; \\
EM RES101 Ion Beam Miller; DCM 3D Measuring \\
Microscope & \\
\hline Maney Publishing & Booth 105 \\
www.maney.co.uk &
\end{tabular}

Journals; Books; E-Journal Collections

MANTIS Deposition Ltd.

Booth 323

www.mantisdeposition.com

Deposition Systems; Nanoparticle Source;

Sputtering Systems

MBraun, Inc.

Booth 421

Www mbraunusa com

Gloveboxes; Gas Purification Systems

Vacuum Systems; Solvent

MDC Vacuum Products, LLC

www.mdcvacuum.com

Flanges and Fittings; Valves; Viewports and

Glass Components; Feedthroughs; Motion and

Manipulation Instruments; Thin Film Deposition;

Custom Engineering; Gas Delivery Systems

Metrohm USA, Inc.

Booth 625

Www.metrohmusa.com

Electro Chemical Systems; Impedance

Characterizations; Sensors; EQCM

\section{METTLER TOLEDO}

Booth 707

WwW.mt.com

MP90; DSC1; TGA/DSC1

Micro Materials Limited

wWw.micromaterials.co.uk

NanoTest System; MicroTest System

Micro Photonics Inc.

WWW.microphotonics.com

SAXS; X-Ray Diffraction; X-Ray CT

\section{Microfluidics}

WWW.microfluidicscorp.com

Micromeritics Instrument Corp.

www.micromeritics.com

Materials Characterization Instrumentation; Particle

Size/Shape Instrumentation; Laboratory Services

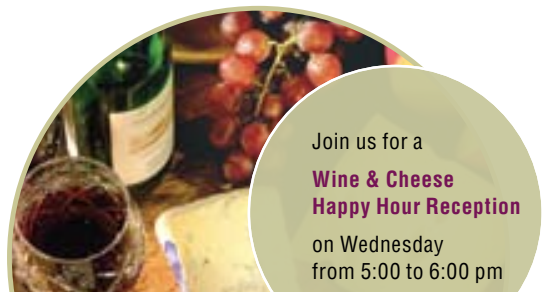

MikroMasch USA

Booth 223

www.spmtips.com

Silicon Probes; Test Structure

MMR Technologies, Inc.

Booth 1003

www.mmr.com

Hall Effect Measurement Systems; Seebeck Effect

Measurement Systems; Variable Temperature

Microprobe Systems

\section{Montana Instruments}

Booth 520

Corporation

wWw.montanainstruments.com

Cryostation

\section{MTI Corporation}

www.mtixtl.com

RTP Furnace; High Pressure Furnace; Battery

Research Equipment

\section{Nagoya University}

www.nagoya-microscopy.jp

Electron Microscope Analysis

\section{nano tech 2011}

www.nanotechexpo.jp/en

Information on World's Largest Nanotechnology

Show; Market Data on nano tech Japan

NanoAndMore USA Inc.

Booth 610

WWW.nanoandmore.com

AFM Probes; Digital Optical Microscopes

Digital Holographic Microscopes

Nanofactory Instruments AB

Booth 704

WWW.nanofactory.com

In situ TEM/SPM; AFM-TEM Systems

TEM Nanoindentation

\section{Nanonics Imaging Ltd.}

www.nanonics.co.il

NSOM; AFM; AFM/Raman

NanoSight
WWW.nanosight.com

\section{Nanosurf AG}

WWW.nanosurf.com

FlexAFM; LensAFM

Nanotec Electrónica S.L.

www.nanotec.es

Full Mode Cervantes AFM; Dulcinea SPM Contro

Unit; WSxM Free SPM Software

\section{Nanounity}

Booth 709

www.nanounity.com

Atomic Force Microscope; Scanning Ion Conducting

Microscope; AFM Probes

\section{Nanovea}

Booth 1029

www.nanovea.com

Profilometers; Nano Mechanical Testers; Tribometers

\section{National Nanotechnology}

Booth 224

Infrastructure Network

www.nnin.org

Nanofabrication; Nanotechnology

National Reconnaissance Office

Booth 220

dii.westfields.net

Research \& Development Funding; Advance

Technology; Remote Sensing

\section{Nature Publishing Group}

Booth 118

www nature.com

Medical \& Scientific Journals

\section{NBM Design, Inc.}

www.nbmdesign.com

Pulsed Laser Deposition; Custom Systems:

Deposition Technology

\section{Neocera, LLC}

www.neocera.com

Pulsed Laser Deposition; Pulsed Electron

Deposition; Laser MBE Systems
Netzsch Instruments

Booth 924

\section{North America, LLC}

www.e-thermal.com; www.netzsch.com

Thermal Analysis; Thermal Conductivity; Thermal

Expansion; Calorimetry

NIST

Booth 216

WWW.nist.gov/srm

Standard Reference Materials; Data and Calibration

Services

NIST, Center for Nanoscale

Booth 217

Science and Technology

www.cnst.nist.gov

Nanoscale Research Program; Nanofabrication

Facility; User Facility

NIST, Technology Innovation

Booth 219

Program

www.nist.gov/tip

Research Funding; Government Funding;

Advanced Materials

Nor-Cal Products, Inc.

www.n-c.com

Vacuum Components \& Chambers; Flanges \&

Fittings; Valves

nPoint, Inc.

Booth 607

www.npoint.com

Nanopositioning Systems; AFM Upgrades;

Control Electronics

NT-MDT Co.

Booth 211

www.ntmdt.com

Scanning Probe Microscopes; Probe

Nanolaboratories; Cantilevers, Calibrations,

Gratings, Test Samples for SPM

Olympus America Inc.

wWw.olympusamerica.com

Inverted Metallographs; Upright Microscopes;

Stereo Microscopes; Filter Inspection

Omicron Nanotechnology USA Booth 317

www.omicron-instruments.com

UHV SPM; Surface Science Instrumentation; MBE

omt optische messtechnik gmbh Booth 611

www omt-instruments com

TFA Reflectometer; TFA Ellipsometer; Light Sources

OriginLab Corporation

Booth 616

www.originlab.com

Origin 8.5 Software

Oxford University Press

Booth 126

www.oup.com/us

Books; Journals

\section{Pan Stanford Publishing \&}

Independent Publishers Group

Booth 123

www.ipgbook.com

Books

PANalytical Inc.

www.panalytical.com

Booth 1000

X-ray Diffraction (XRD); Computed Tomography

(CT); Small Angle X-ray Scattering (SAXS); X-ray

Fluorescence (XRF)

Park Systems Inc.

Booth 410

www parkafm com

AFM Products; AFM Mode Options

Particle Sizing Systems

www.pssnicomp.com

AccuSizer SPOS; Archimedes; Nicomp DLS

Pascal Technologies Inc.

www.pascaltechnologies.com

Vacuum Systems; Vacuum Equipment

Leak Detectors

Photon Technology

International, Inc.

www.pti-nj.com

Steady State Fluorescence; Time-Resolved

Fluorescence; NIR Luminescence
Booth 1310

Booth 810

Booth 729 


\section{Plasma-Therm LLC}

Booth 609

www plasmatherm com

Semiconductor Manufacturing Equipment

Plasmaterials, Inc.

Booth 320

PWw plasmaterials com

Sputtering Targets; Backing Plates; Evaporation Materials

Plasmionique Inc.

Booth 820

www.plasmionique.com

PVD Systems; Plasma Reactors; Plasma \& PVD

Sources

\section{Princeton Instruments}

Booth 718

www.princetoninstruments.com

Spectroscopy Systems; CCD Cameras;

Spectrometers

Princeton Scientific Corp.

www.princetonscientific.com

Metal Crystals; Laser Components; Superconductor Wafers; Optical Components

\begin{tabular}{lc} 
Process Materials, Inc. & Booth $\mathbf{8 0 3}$ \\
www.processmaterials.com & \\
Sputtering Targets; Evaporation Materials; & \\
Cylindrical Cathode & \\
\hline Protochips, Inc. & Booth $\mathbf{6 0 2}$ \\
Www.protochips.com & \\
TEM and SEM Accessories; In situ Science
\end{tabular}

TEM and SEM Accessories; In situ Science

PVD Products, Inc.

Booth 1201

Www pvdproducts com

Sputter Systems; Sputter Sources; Pulsed Lase

Deposition Equipment

QD Solution Co., Ltd.

Booth 1207

QWw

Quantum Dot; LED; Display

Quantum Design, Inc.

www.qdusa.com

Physical Property and Magnetic Property

Measurement Systems

Radiant Technologies, Inc.

www.ferrodevices.com

Ferroelectrics; Multiferroics; Piezoelectrics

Raith USA, Inc.

Booth 923

www. raith com

E-Beam Lithography; Nanotechnology

RBD Instruments, Inc.

www.rbdinstruments com

Booth 909

IG2 2kV Backfill Ion Source Package; Compact

Auger Analyzer; 9103 USB Graphing Picoammeter;

UVB-100 Water Desorption System; PC Upgrades for Surface Analysis Instruments

Refining Systems, Inc.

Booth 1009

www refiningsystems com

Sputtering Targets; Evaporation Materials;

Wires and Tubing

Renishaw Inc.

Booth 324

www.renishaw.com

Raman Microscopes; Spectrometers

Research and PVD

Materials Corporation

www.pvdmaterials.com

PVD Materials; Sputtering Targets; Evaporation

Sources

RHK Technology, Inc.

Booth 603

www.rhk-tech.com

SPM Universal Controls; UHV STM; UHV AFM/STM

Rigaku Americas Corporation Booth 524

www.rigaku.com

X-ray Diffraction Systems; Small Angle X-ray

Scattering Systems
RKI Instruments, Inc.

Booth 819

www.rkiinstruments.com

Photo-electron Spectroscopy

Rocky Mountain Vacuum Tech, Inc. Booth 322

www.rmvac.com

Vacuum Systems; Vacuum Components

RSC Publishing
www.rsc.org/publishing
Journals; Books

Booth 114

SAGE Publications

Booth 121

WwW.sagepub.com

Books; Journals

\section{Scientific Instruments, Inc.}

www. scientificinstruments com

9700 Controller; SCM10; Diodes

Seki Technotron USA

www.sekicvdsolutions.com

Microwave Plasma CVD Systems; Hot Filament

CVD Systems; Plasma CVD Systems

Semiconsoft, Inc.

wWw.semiconsoft.com

MProbe Thin-film Measurement System

TFCompanion Software

Semicore Equipment Inc.

www.semicore.com

Sputtering Systems; Evaporation Systems; Custom

PVD Systems \& Components

Silicon Sense, Inc.

Booth 832

www.siliconsense.com

Silicon Wafers; Foundry Services

\section{Solartron Analytical (AMETEK)}

www.solartronanalytical.com

ModuLab MTS; 1260 Impedance Analyzer

Cryostats \& Furnaces

South Bay Technology, Inc.

www.southbaytech.com

Ion Beam Sputter Deposition; Reactive Ion Etcher;

Plasma Cleaner/Trimmer

SouthWest NanoTechnologies Inc. Booth 705

wwW.swentnano.com

Single-wall Carbon Nanotubes (CNT); Specialty

Multiwall CNT; CNT-based Printable Inks; CNT-based Smart Fabrics

SPECS Surface Nano Analysis GmbH Booth 702

wWw.specs.de

PHOIBOS Energy Analyzer; SPM 150 Aarhus:

JT-STM; FE-LEEM P90

SPEX SamplePrep LLC

Booth 1008

www spexsampleprep.com

Mixer/Mills; Freezer/Mills; X-Press

SPI Supplies, Division of

Booth 1202

Structure Probe, Inc.

www.2spi.com

Plasma Etchers; TEM Membrane Windows

Vacuum System Consumables

Spire Biomedical, Inc.

www.spirecorp.com

Thin Film; Biomedical; Nano-materials; Coating

Surface Treatment

\section{Springer}

Booth 101

www.springer.com

Books; Journals; Periodicals

STAIB Instruments, Inc.

www staibinstruments com

RHEED; Spectrometer; Surface Analysis

STAR Cryoelectronics

www.starcryo.com

X-ray Microanalysis; SQUID Sensors; Foundry Services

Strem Chemicals, Inc.

www.strem.com

Nanomaterials; CVD and ALD Precursors; Chemicals
Structured Materials Industries, Inc. Booth 720

WWW.structuredmaterials.com

MOCVD Systems; ALD Systems; Thin Film

Deposition Components

Super Conductor Materials, Inc.

Booth 618

www.scm-inc.com

Sputtering Targets; Evaporation Materials

Crucible Liners

SVT Associates, Inc.

Booth 420

www svta com

MBE; PVD; PLD; ALD; Thin Film Deposition

Taylor \& Francis Group-CRC Press Booth 104

www.taylorandfrancis.com

Books; Journals; Online Products

TCl America

Booth 818

www.tciamerica.com

Specialty Chemicals; Conductive Polymers:

Electroluminescent Materials

TDK-Lambda Americas Inc.

www.us.tdk-lambda.com/hp

Booth 904

Genesys ${ }^{T M}$ Programmable AC/DC Power Supplies

Ted Pella, Inc.

Booth 516

www.tedpella.com

Vacuum Coaters; Calibration; Microscopy Sample

Preparation \& Supplies/Accessories

TEMwindows.com

Booth 1304

www temwindows.com

TEM Windows; Nanoparticle Separation Devices

TeraView Ltd.

www.teraview.com

THz Imaging and Spectroscopic Systems

The Mellen Company, Inc.

Booth 1010

www.mellencompany.com

Furnaces; Controls; Heating Elements

Thermionics Vacuum Products

Booth 409

www.thermionics.com

Sample Handling; Thin Film Deposition Equipment

Valves

Thermo Scientific

Booth 422

www.thermoscientific.com

Surface Analysis; Raman/InfraRed; Microanalysis

Thinky USA, Inc.

Booth 728

www.thinky.co.jp/us

Non-Vacuum Mixer/Vacuum Mixer

\section{Toho Technology}

www.tohotechnology.com

FLX-2320-S Thin Film Stress Measurement System; FP Series Stylus Profilers

TSI Inc.

Booth 735

www.tsi.com

Research Optical Particle Sizer; Water-Based

Condensation Particle Counters

Trek, Inc.

Booth 711

www.trekinc.com

High-Voltage Amplifiers; Contacting/Noncontacting

Electrostatic Voltmeters; Surface Charge/Resistance Measurement

Trion Technology, Inc.

Booth 829

www.triontech.com

Reactive Ion Etcher (RIE); Plasma Enhanced

Chemical Vapor Deposition (PECVD); Stripping Systems

ULVAC Technologies, Inc.

Booth 713

www.ulvac.com

Seebeck Coefficient; RTP Annealing Furnace;

Thermal Diffusivity

VAT, Inc.

Booth 801

WwW vatvalve com

Vacuum Valves; Controllers; Transfer Valves

Veeco Instruments Inc.

(see Bruker Nano Surfaces Business)

Booth 300 
VG Scienta, Inc.

WwW.vgscienta.com

Surface Analysis Systems \& Components;

UHV Systems \& Components; Valves; Sample

Manipulators

\section{Virginia Semiconductor, Inc.}

Booth 311

www.virginiasemi.com

Silicon Wafers; SOI Wafers; Ultrathin Wafers

Wafer World Inc.

Booth 905

www waferworld com

Silicon Wafers; GaAs Wafers; Clean Room Packaging

Wiley/Wiley-VCH

Booth 120

WWW.wiley.com

Books; Journals; Databases

Williams Advanced Materials Booth 1309

www.williams-adv.com

Specialty Inorganic Chemicals; Evaporation

Materials; Sputtering Targets
WITec Instruments Corp.

www.witec-instruments.com

Booth 613

Confocal Raman Microscopy; Scanning Near-Field; Raman AFM

J.A. Woollam Company, Inc. Booth 1004 www.jawoollam.com

Spectroscopic Ellipsometers; Measurement Services

WV Nano Initiative

Booth 1302

wvnano.wvu.edu

Nanoscience Research; Work Force Development;

Shared Facilities

X-Ray Optical Systems, Inc. Booth 824

www.Xos.com

Polycapillary Optics; Doubly-curved Crystal;

X-Beam-X-ray Source with Optics

XEI Scientific, Inc

Booth 1028

www.evactron.com

inators for

De-contaminators for SEMs; De-c
TEMs; Lower-Power RF Supply
Yeagle Technology Inc.

Booth 807

www.ytionline.com

Vacuum Systems; On-site Service; Vacuum

Components

\section{Zeta Instruments}

www.zeta-inst.com

3D Microscope; 3D Profile Measurements;

Film Thickness Measurements

Zurich Instruments Ltd.

www.zhinst.com

HF2LI Lock-in Amplifier; HF2IS Impedance

Spectroscope

\section{Zygo Corporation}

www.zygo.com

High Precision Measurement Systems; Optics;

Optical Design/Assembly

\section{Handbook of Modern Ion Beam Materials Analysis}

\section{Second Edition}

NOW AVAILABLE

The most comprehensive database on ion beam analysis ever published- revised and updated from the popular handbook released in 1995!

\section{EDITORS}

Yongqiang Wang \& Michael Nastasi

- Written and compiled by over 30 leading authorities in the field of ion beam analysis

- Important reference tool for technicians, students and professionals

- A must for all accelerator labs

- Excellent introduction to the fundamentals and lab practices of ion beam analysis

- Useful as a teaching text for undergraduate senior or first-year graduate students

- For libraries, the most recent and comprehensive collection of nuclear and atomic data for the applications of ion beam materials analysis

- DVD includes bonus info-Ion Beam Analysis Nuclear Data Library (IBANDL) and GUPIX Subroutines (CSA and YLS) for X-ray Database

TWO VOLUME PRINT SET + DVD OF APPENDICES

Order Code: IBH-2 • ISBN: 978-1-60511-217-6

$\$ 200.00$ MRS Members \$250.00 Nonmembers

VOLUME 1-PRINT CHAPTERS (441 PAGES) + DVD OF APPENDICES Order Code: IBH-2a • ISBN: 978-1-60511-215-2

\$125.00 MRS Members \$150.00 Nonmembers

VOLUME 2-PRINT APPENDICES ONLY (370 PAGES)

Order Code: IBH-2b • ISBN: 978-1-60511-216-9

$\$ 125.00$ MRS Members \$ \$150.00 Nonmembers

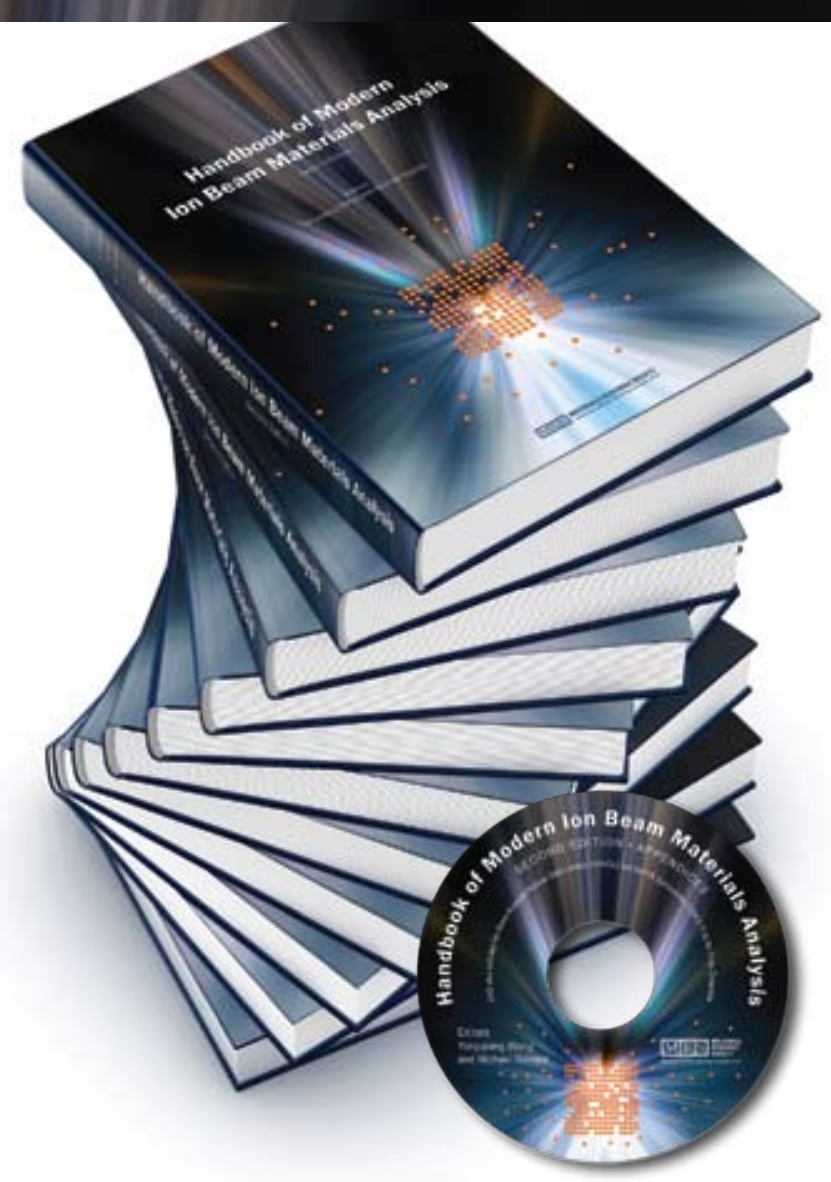

ORDER AT WWW.MRS.ORG/IBH2

MIRS MATERIALS RESEARCH SOCIETY, Customer Services, 506 Keystone Drive, Warrendale, PA 15086 USA • Tel 724.779.3003・info@mrs.org 
FOCUS ISSUE

Journal of Materials Research

August 2011

\section{Advances in Thermoelectric Materials}

Thermoelectric materials exhibit the novel attribute that they can convert thermal energy (heat) directly into electricity as a solid-state conversion process with no moving parts. The thermoelectric conversion efficiency is directly proportional to the Carnot efficiency of a heat engine operating between the two temperatures. Thermoelectric materials can also behave as solid-state refrigerators via the Peltier effect in these materials. Recently, there have been significant advances in direct thermalto-electrical energy conversion materials and this has generated increased interest in the field. JMR will publish a focused issue on Thermoelectric Materials in August 2011. This issue will capture some of the most important recent advances in fundamental materials design and resulting thermoelectric properties. The issue will also include several review articles that will highlight the important developments and various directions of the field over the last few years.

This focused issue will include a combination of new theoretical ideas, new materials and new device concepts in the field. It will focus on novel materials, various methods of materials processing and synthesis along with technologies and applications related to direct thermal-to-electric energy conversion and cooling.

Topics to be addressed include, but are not limited to, the following:

- Oxides and other materials with strong electron correlation

- Theoretical guidance to high efficiency thermoelectric energy conversion

- New and emerging technologies for TE power conversion

- High efficiency bulk TE materials

- Composite and nano-composite thermoelectrics

- Thermoelectrics related to harvesting solar energy

- Low dimensional aspects of TE materials

- Synthetic strategies for preparing novel materials and compounds

- Role of spark plasma sintering techniques for TE materials

- Processing of bulk and thin film nanostructured materials

- Materials property measurement and new measurement techniques

- Design, performance testing, fabrication and processing of energy conversion devices

- Applications and new directions in thermal energy conversion

- Advances in materials property measurements and characterization

\section{GUEST EDITORS}

Terry M. Tritt, Clemson University, USA

Harald Böttner, Fraunhofer Institut für Physikalische Messtechnik IPM

SUBMISSION DEADLINE: DECEMBER 15, 2010. Please select "Focus Issue: Advances in Thermoelectric Materials" as the manuscript type when submitting.

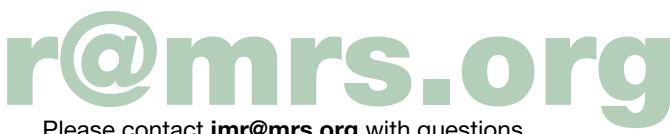

Please contact jmr@mrs.org with questions.
FOCUS ISSUE

Journal of Materials Research

September 2011

\section{Nanowires: Fundamentals and Applications}

Research on nanowires encompasses fundamental issues in crystal growth and the scaling of materials properties to molecular dimensions, and work on possible applications of nano-scale single crystal assemblies in advanced devices. The goal of this JMR focus issue is to summarize leading research performed by the broad, multidisciplinary community of nanowire researchers to provide a review of 1 ) the current state of the field and 2) the opportunities for future high-impact science and technology related to semiconductor nanowires.

Contributed papers are solicited on the following topics:

- Top-down and bottom-up assembly of nanowire arrays

- Catalyzed versus non-catalyzed nanowire growth

- Catalyst materials selection

- Vapor, solution, and template mediated nanowire growth

- Thermodynamics and kinetics of nanowire nucleation and growth

- Crystallographic and topological orientation control

- Axial and core-shell heterostructures - synthesis and properties

- Limits to nanowire area and length scaling

- Size-dependent electronic, optical and mechanical properties

- Surface passivation and functionalization of nanowires

- Dopant incorporation and activation

- Metal/semiconductor nanowire contact formation

- Applications of nanowires in future devices

\section{GUEST EDITORS}

Paul C. McIntyre, Stanford University, USA

Volker Schmidt, Max Planck Institute, Halle, Germany

\section{PRINCIPAL EDITORS}

Tom Picraux, Los Alamos National Laboratory, USA

Nathaniel Quitoriano, McGill University, Canada

Heike Riel, IBM Research, Switzerland

Claes Thelander, Lund University, Sweden

Carl Thompson, Massachusetts Institute of Technology, USA

SUBMISSION DEADLINE: JANUARY 28, 2011.

Please select "Focus Issue: Nanowires: Fundamentals

and Applications" as the manuscript type when submitting.

\section{MANUSCRIPT SUBMISSION FOR JMR FOCUS ISSUES}

To be considered for a JMR focus issue, new and previously unpublished results significant to the development of this field should be presented. The manuscripts must be submitted via the JMR electronic submission system. Manuscripts submitted after the submission deadline will not be considered for the issue due to time constraints on the review process. Submission instructions may be found at www.mrs.org/jmr_instructions. All manuscripts will be reviewed in a normal but expedited fashion. Papers submitted by the deadline and subsequently accepted will be published in the Focus Issue. Other manuscripts that are acceptable but cannot be included in the issue will be scheduled for publication in a subsequent issue of $J M R$. 

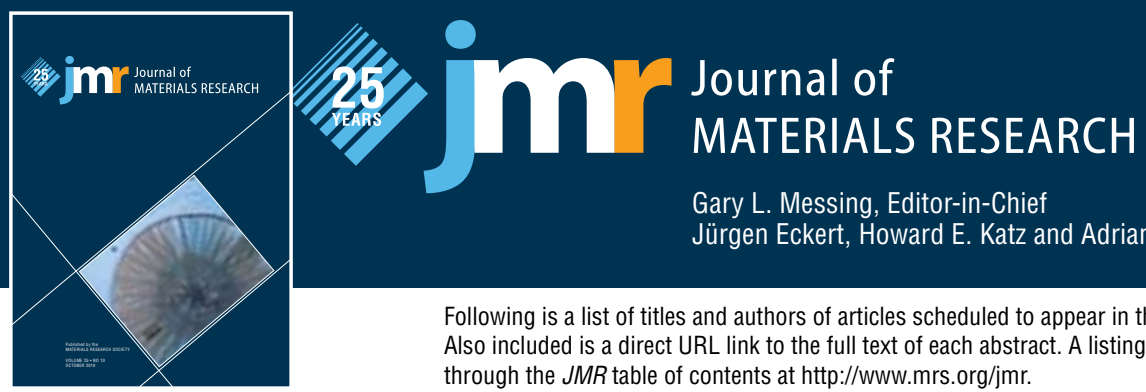

VOLUME $25 \cdot$ NO 12 • DECEMBER 2010

Gary L. Messing, Editor-in-Chief

Jürgen Eckert, Howard E. Katz and Adrian Mann, Associate Editors

Following is a list of titles and authors of articles scheduled to appear in the upcoming issue of the Journal of Materials Research Also included is a direct URL link to the full text of each abstract. A listing of all JMR abstracts from 1986 to the present is available through the JMR table of contents at http://www.mrs.org/jmr

\section{INVITED FEATURE PAPER}

Nanoscale strength distribution in amorphous versus crystalline metals

C.E. Packard, O. Franke, E.R. Homer, C.A. Schuh

Massachusetts Institute of Technology

http://www.mrs.org/jmr_10_0299

\section{ARTICLES}

Quasi-static and dynamic deformation behaviors of in situ Zr-based bulk-metallic-glass-matrix composites J.W. Qiao, ${ }^{1}$ P. Feng, ${ }^{2}$ Y. Zhang, ${ }^{1}$ Q.M. Zhang, ${ }^{2}$ P.K. Liaw, G.L. Chen

${ }^{1}$ University of Science and Technology Beijing, ${ }^{2}$ Beijing Institute of Technology, ${ }^{3}$ University of Tennessee http://www.mrs.org/jmr_10_0289

In situ high-energy x-ray diffraction observation of structural evolution in a Ti-based bulk metallic glass upon heating

N. Zheng, ${ }^{1}$ G. Wang, ${ }^{1}$ L.C. Zhang, ${ }^{2}$ M. Calin ${ }^{1}$ M. Stoica,

G. Vaughan, ${ }^{3}$ N. Mattern, ${ }^{1}$ J. Eckert ${ }^{1,4}$

${ }^{1}$ IFW Dresden, Institute for Complex Materials,

${ }^{2}$ The University of Western Australia, ${ }^{3}$ European

Synchrotron Radiation Facilities, ${ }^{4}$ Technical University

of Dresden

http://www.mrs.org/jmr_10_0298

Abnormal spalling phenomena in the $\mathrm{Sn}-0.7 \mathrm{Cu} / \mathrm{Au} / \mathrm{Ni}$ SUS304 interfacial reactions

Shih-kang Lin, ${ }^{1}$ Kuen-da Chen, ${ }^{1}$ Hao Chen, ${ }^{1}$

Wei-kai Liou ${ }^{2}$ Yee-wen Yen

${ }^{1}$ National Taiwan University of Science and Technology,

${ }^{2}$ Lunghwa University of Science and Technology

http://www.mrs.org/jmr_10_0305

Fiber metallic glass laminates

B.A. Sun, ${ }^{1,2}$ K.P. Cheung, ${ }^{2}$ J.T. Fan, ${ }^{2}$ J. Lu, ${ }^{2}$ W.H. Wang ${ }^{1}$ Institute of Physics, Chinese Academy of Sciences,

${ }^{2}$ The Hong Kong Polytechnic University

http://www.mrs.org/jmr_10_0291

Transition from a punched-out dislocation to a slip dislocation revealed by electron tomography Masaki Tanaka, ${ }^{1}$ Grace S. Liu, ${ }^{2}$ Tomonobu Kishida, ${ }^{1}$ Kenji Higashida, ${ }^{1}$ lan M. Robertson ${ }^{2}$

${ }^{1}$ Kyushu University, ${ }^{2}$ University of Illinois

http://www.mrs.org/jmr_10_0308

Determining engineering stress-strain curve directly from the load-depth curve of spherical indentation test

Baoxing Xu, ${ }^{1} \mathrm{Xi} \mathrm{Chen}^{1,2,3}$

${ }^{1}$ Columbia University, ${ }^{2}$ Hanyang University,

${ }^{3}$ Xi'an Jiaotong University

http://www.mrs.org/jmr_10_0310

Error estimation of nanoindentation mechanical properties near a dissimilar interface via finite element analysis and analytical solution methods Y. Zhao, T.C. Ovaert

University of Notre Dame

http://www.mrs.org/jmr_10_0295

Co alloying and size effects on solidification and

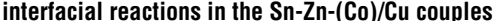
Yu-chih Huang, Sinn-wen Chen

National Tsing Hua University

http://www.mrs.org/jmr_10 0314
First-principles study of the structural and elastic properties of $\mathrm{Ti}_{5} \mathrm{Si}_{3}$ with substitutions $\mathrm{Zr}, \mathrm{V}, \mathrm{Nb}$, and $\mathrm{Cr}$

Hui-Yuan Wang, Wen-Ping Si, Shi-Long Li, Nan Zhang,

Qi-Chuan Jiang

Jilin University

http://www.mrs.org/jmr_10_0293

Enhanced hardness of CrAISiN/W, N superlattice coatings deposited by DC magnetron sputtering YZ Tsai, J.G. Duh

National Tsing-Hua University

http://www.mrs.org/jmr_10_0296

Structural stability and Raman scattering of $\operatorname{InN}$ nanowires under high pressure

L.D. Yao, ${ }^{1}$ S.D. Luo, ${ }^{1}$ X. Shen, ${ }^{1}$ S.J. You, ${ }^{1}$ L.X. Yang, S.J. Zhang, ${ }^{1}$ S. Jiang, ${ }^{2}$ Y.C. Li, ${ }^{2}$ J. Liu, ${ }^{2}$ K. Zhu, ${ }^{1}$ Y.L. Liu,

W.Y. Zhou, ${ }^{1}$ L.C. Chen, ${ }^{1}$ C.Q. Jin, ${ }^{1}$ R.C. Yu, ${ }^{1}$ S.S. Xie ${ }^{1}$

${ }^{1}$ Beijing National Laboratory for Condensed Matter

Physics, Institute of Physics, Chinese Academy of

Sciences, 2 Institute of High Energy Physics, Chinese

Academy of Sciences

http://www.mrs.org/jmr_10_0290

Metastable nanosized diamond formation from

C-H-O fluid system

S.K. Simakov

St. Petersburg University

http://www.mrs.org/jmr_10_0303

Amorphization of nanocrystalline 3C-SiC irradiated with $\mathrm{Si}^{+}$ions

W. Jiang, ${ }^{1}$ H. Wang, ${ }^{2}$ I. Kim, ${ }^{2}$ Y. Zhang, ${ }^{1}$ W.J. Weber ${ }^{3,4}$ ${ }^{1}$ Pacific Northwest National Laboratory, ${ }^{2}$ Texas A\&M University, ${ }^{3}$ University of Tennessee, ${ }^{4}$ Oak Ridge

National Laboratory

http://www.mrs.org/jmr_10_0311

Irradiation-induced defect clustering and

amorphization in silicon carbide

William J. Weber, ${ }^{1,2}$ Fei Gao ${ }^{3}$

${ }^{1}$ University of Tennessee, ${ }^{2}$ Oak Ridge National Laboratory,

${ }^{3}$ Pacific Northwest National Laboratory

http://www.mrs.org/jmr_10_0292

Silicon nitride nanoceramics densified by dynamic grain sliding

Mathias Herrmann, ${ }^{1}$ Zhijian Shen, ${ }^{2}$ Ingrid Schulz,

Jianfeng $\mathrm{Hu}^{2}$ Bostjan Jancar

${ }^{1}$ Fraunhofer Institute of Ceramic Technologies and Sintered Materials, ${ }^{2}$ Arrhenius Laboratory Stockholm University, ${ }^{3}$ Institut für Werkstoffwissenschaft,

Technical University of Dresden, ${ }^{4}$ Jozef Stefan Institute http://www.mrs.org/jmr_10_0313

Nucleation and growth rate influence on

microstructure and critical currents of TFA- $\mathrm{YBa}_{2} \mathrm{Cu}_{3} \mathrm{O}$ under low-pressure conditions

H. Chen ${ }^{1,2}$ K. Zalamova, ${ }^{2}$ A. Pomar, ${ }^{2}$ X. Granados, ${ }^{2}$

T. Puig, ${ }^{2} X$. Obradors

${ }^{1}$ Tsinghua University, ${ }^{2}$ Institut de Ciència de Materials de Barcelona (ICMAB-CSIC)

http://www.mrs.org/jmr_10_0302

Effect of $\mathrm{BaCu}\left(\mathrm{B}_{2} \mathrm{O}_{5}\right)$ on the sintering temperature and microwave dielectric properties of

$\mathrm{Ba}\left(\mathrm{Nd}_{\mathrm{O}} \mathrm{Bi}\right) \mathrm{Ti}, \mathrm{O}_{12}$ ceramics

Yi Zeng, ${ }^{1}$ Hong Wang, ${ }^{1}$ Huanfu Zhou ${ }^{2}$

${ }^{1}$ Xi'an Jiaotong University, ${ }^{2}$ Guilin University of Technology http://www.mrs.org/jmr_10 0309

Revealing the mechanism of the early stages of $\mathrm{Ni}-\mathrm{W}$ RABiTS oxidation

Andrey V. Blednov, ${ }^{1}$ Oleg Yu. Gorbenko,

Dmitriy P. Rodionov, ${ }^{2}$ Andrey $R$. Kau

${ }^{1}$ Moscow State University, ${ }^{2}$ Institute of Metal Physics,

Ural Division, Russian Academy of Sciences

http://www.mrs.org/jmr_10 0312

Microstructure and electrical properties in threecomponent $\left(\mathrm{Al}_{2} \mathrm{O}_{3}-\mathrm{TiO}_{2}\right) /$ polyimide nanocomposite films

Jun-Wei Zha, ${ }^{1,3}$ Ben-Hui Fan, ${ }^{1}$ Zhi-Min Dang, ${ }^{1}$ ShengTao Li, ${ }^{2}$ George Chen

${ }^{1}$ Beijing University of Chemical Technology, ${ }^{2} X i$ 'an Jiaotong University, ${ }^{3}$ University of Southampton

http://www.mrs.org/jmr_10_0306

Enhanced photocatalytic activity of (Mo, C)-codoped anatase $\mathrm{TiO}_{2}$ nanoparticles for degradation of methy orange under simulated solar irradiation

Pengyu Dong, ${ }^{1}$ Bin Liu, ${ }^{1}$ Yuhua Wang, ${ }^{1}$ Huanhuan Pei, Shu Yin²

'Lanzhou University, ${ }^{2}$ Tohoku University

http://www.mrs.org/jmr_10_0307

Spectroscopic analysis of tungsten oxide thin films Felicia S. Manciu, Jose L. Enriquez, William G. Durrer, Young Yun, Chintalapalle V. Ramana, Satya K. Gullapalli University of Texas at El Paso

http://www.mrs.org/jmr_10_0294

Preparation and characterization of the

defect-conductivity relationship of Ga-doped $\mathrm{ZnO}$ thin films deposited by non-reactive radio

frequency-magnetron sputtering

M. Lalanne, J.M. Soon, A. Barnabé, L. Presmanes, I. Pasquet, Ph. Tailhades

Université de Toulouse UPS-INP-CNRS Institut Carnot CIRIMAT

http://www.mrs.org/jmr_10_0300

Improved dehydrogenation of $\mathrm{LiBH}_{4}$ supported on nanoscale $\mathrm{SiO}_{2}$ via liquid phase method

X.Y. Chen ${ }^{1}$ Y.H. Guo,${ }^{1}$ L. Gao ${ }^{1}$ X.B. Y Yu ${ }^{1,2}$

${ }^{1}$ Fudan University, ${ }^{2}$ University of Wollongong http://www.mrs.org/jmr_10_0301

Synthesis and tunable photoluminescence of $\mathrm{NaYF}_{4}:$ Eu/Ba nanocrystals

Guofeng Wang, Qing Peng

Tsinghua University

http://www.mrs.org/jmr_10_0297

Preparation of Culn $\left(\mathrm{S}_{x} \mathrm{Se}_{1-\mathrm{x}}\right)$, thin films with tunable band gap by controlling sulfurization temperature of CulnSe

Guangjun Wang, Gang Cheng, Binbin Hu, Xiaoli Wang Shaoming Wan, Sixin Wu, Zuliang Du

Henan University

http://www.mrs.org/jmr_10_0304

For general manuscript preparation and submission visit wWW.mrs.org/jmr instructions

This preliminary listing of titles is tentatively scheduled to appear in the Journal of Materials Research at the time MRS Bulletin is published. Late schedule changes before JMR is printed may result in articles being added or deleted. The Materials Research Society regrets any inconvenience that may result from schedule changes.

Copyright ( 2010 by Materials Research Society. All rights reserved. ISSN: 1066-2375. 


\section{Materials Research Society would like to thank the following for their financial support:}

\section{M Company}

ACS Publications

Advanced Diamond Technologies, Inc.

AGD Material Co., Ltd.

Agilent Technologies

AIST-NT, Inc.

AIXTRON AG

Aldrich Materials Science

Alfa Aesar, a Johnson Matthey Company

AlphaMicron, Inc.

American Institute of Physics

American Scientific Publishers

Angstrom Engineering Inc.

Annealsys

ANSTO Institute of Materials Engineering

Apollo Diamond, Inc.

Applied Diamond, Inc.

Applied Materials, Inc.

Applied NanoFluorescence, LLC

Argonne National Laboratory

Asylum Research

BAE Systems

Beneq Oy

Blake Industries Inc.

Boeing Company

Bruker

Bruker Nano Surfaces Business

Cabot Corporation

Cambridge NanoTech Inc.

Cambridge Research Biochemicals

CAMECA

Carl Zeiss AG

Companhia Brasileira de Metalurgia e Mineração

(CBMM) \& Reference Metals Company, Inc.

CEA Marcoule

CEA Saclay

CEOS GmbH

CNRS, National Center for Scientific Research

Corning Incorporated

Covidien

Cree, Inc.

CrysTec GmbH

CSM Instruments Inc.

DCA Instruments, Inc.

Donaldson Company, Inc.

Dow Corning Corporation

DuPont Air Products NanoMaterials, LLC

eBiosciences, Inc.

Element Six

ELMARCO

Evonik Degussa GmbH

FEl Company

Fischione Instruments, Inc.

Fujimi Corporation

G24 Innovations

Gatan, Inc.

GE Global Research
Gelest, Inc.

Genzyme Corporation

Georgia Tech Research Institute

GMZ Energy, Inc.

Group4 Labs, Inc.

Hecus X-Ray Systems GmbH

Helsinki University of Technology

Hitachi High Technologies America, Inc.

Honda Research Institute USA, Inc.

HORIBA Scientific

Huber Diffraktionstechnik GmbH \& Co. KG

Hysitron, Inc.

IBM Almaden Research Center

IBM T.J. Watson Research Center

IBM Zurich Research Laboratory

ICDD (International Centre for Diffraction Data)

Industrial Technology Research Institute,

Photovoltaics Technology Center

INHA University

INRS-EMT, University of Quebec

Institute for Materials Research,

Hasselt University

Institute for Transuranium Elements

Institute fur Eisenhuttenkunde

JEOL Ltd.

JEOL USA, Inc.

JSR Micro, Inc.

K-TEK Nanotechnology

Kleindiek Nanotechnik GmbH

Kurt J. Lesker Company

Lake Shore Cryotronics, Inc.

Lambda Solutions Inc.

Lambda Technologies Inc.

Lawrence Berkeley National Laboratory

Lawrence Livermore National Laboratory

Levitronix

L'OREAL SA

Los Alamos National Laboratory

M. Watanabe \& Co., Ltd.

Mad City Labs Inc.

Maney Publishing

Marlow Industries, Inc.

Medtronic, Inc.

Millipore Corporation

Mitsubishi Chemical Group

MORESCO Corporation

Nanofactory Instruments

Nanonics Imaging Ltd.

National Institute for Materials Science

National Renewable Energy Laboratory (NREL)

National Taiwan University

NETZSCH Instruments N.A. LLC

Ningbo Institute of Materials Technology and

Engineering (NIMTE)

Nokia Corporation

Oak Ridge National Laboratory

Oxford Instruments
PARC (Palo Alto Research Center)

Park Systems

Photon Technology International,Inc.

Plextronics Inc.

Princeton Instruments

Radiant Technologies, Inc.

Raith USA Inc.

Renewable and Sustainable Energy Institute (RAESI)

Renishaw Inc.

Research and PVD Materials Corporation

Riber S.A.

Samsung Electronics Co., Ltd.

Sandia National Laboratories

Seki Technotron Corporation

Semprus Biosciences

Setaram Inc.

Solvay S.A

SPECS Surface Nano Analysis GmbH

Springer

SPS Syntex Inc

Stanford University

Strem Chemicals, Inc.

SuperPower Inc.

SURFACE Systems \& Technologies

$\mathrm{GmbH} \& \mathrm{Co} . \mathrm{KG}$

SwissNeutronics

Tanaka Precious Metals Co., Ltd.

Thermal Technology LLC

Thin Film Electronics ASA

Tohoku University

Tokyo Electron America, Inc.

Tripod Technology Corporation

ULVAC Technologies Inc.

United Solar Ovonic LLC

United Technologies Research Center

Universal Display Corporation

Universal Systems Co. Ltd.

University of Nevada

VAT, Inc.

VSP, an Imprint of Brill

Wiley-VCH Verlag GmbH \& Co.

J.A. Woollam Company, Inc.

World Gold Council

Xerox Corporation

ZT Plus

Zygo Corporation

For more information about the

Corporate Participation Program contact:

Mary E. Kaufold

Manager, Advertising \& Exhibits

Materials Research Society

Tel: 724-779-2755

Cell: 724-996-5683

kaufold@mrs.org 


\section{Premiering January 19, 2011}

\section{NOVA

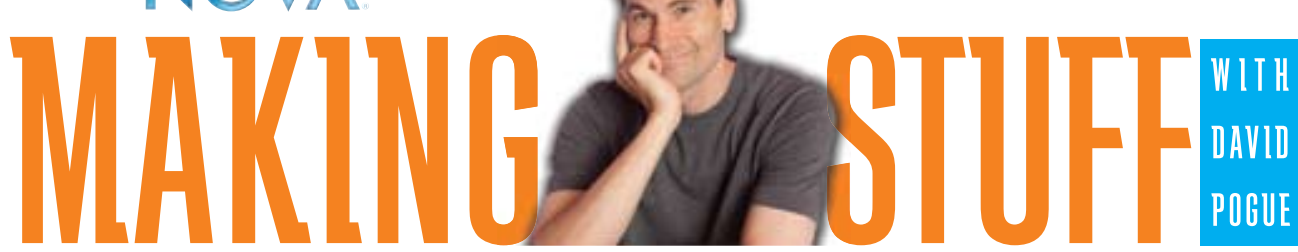

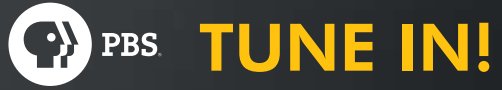

Watch MAKING STUFF with David Pogue on PBS (check local listings)

A four-part series

exploring the materials that will shape the future

MAKING STUFF: Stronger, Smaller, Cleaner, Smarter

Wednesday, January 19 - Stronger

Wednesday, January 26 - Smaller

Wednesday, February 2 - Cleaner

Wednesday, February 9 - Smarter

\section{GET INVOLVED!}

Want to learn more about the series and how you can get involved?

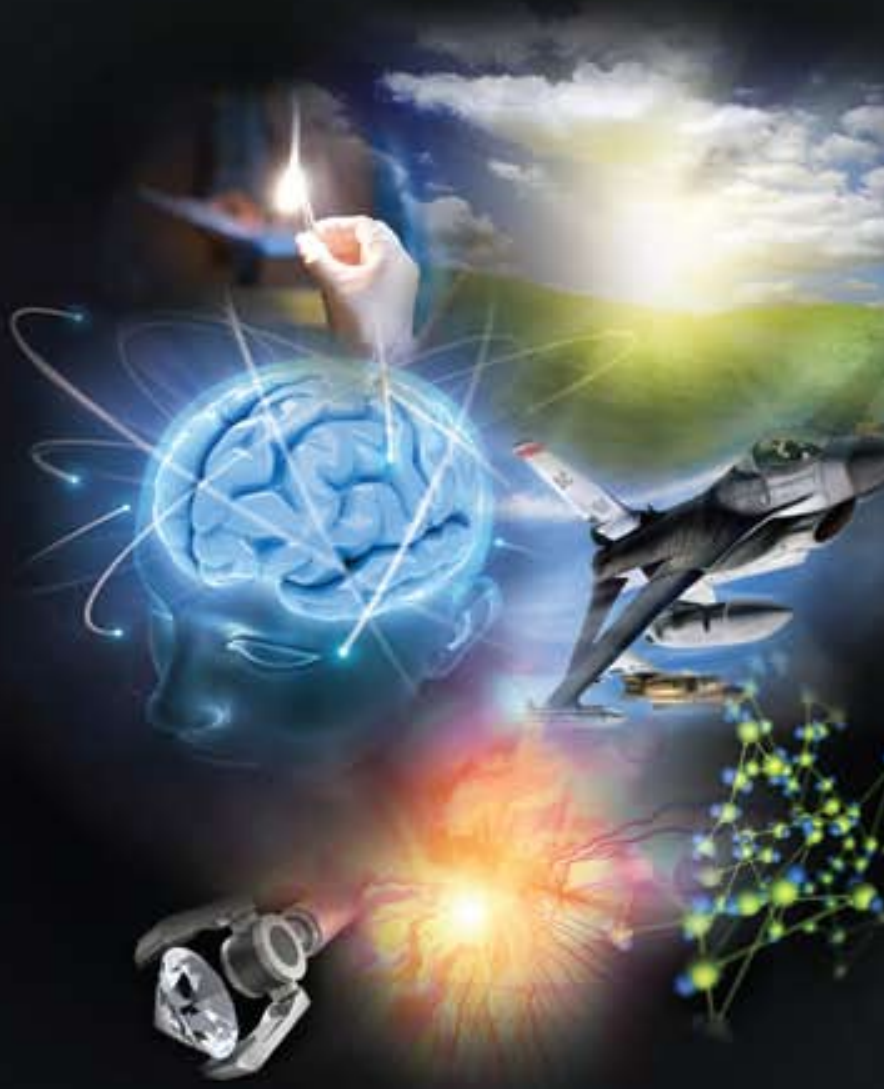

Visit www.mrs.org/makingstuff for details. 
(

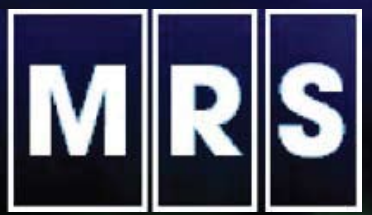

International Union of Materials Research Societies

International Conference on Advanced Materials

IUMRS ICAM 2011
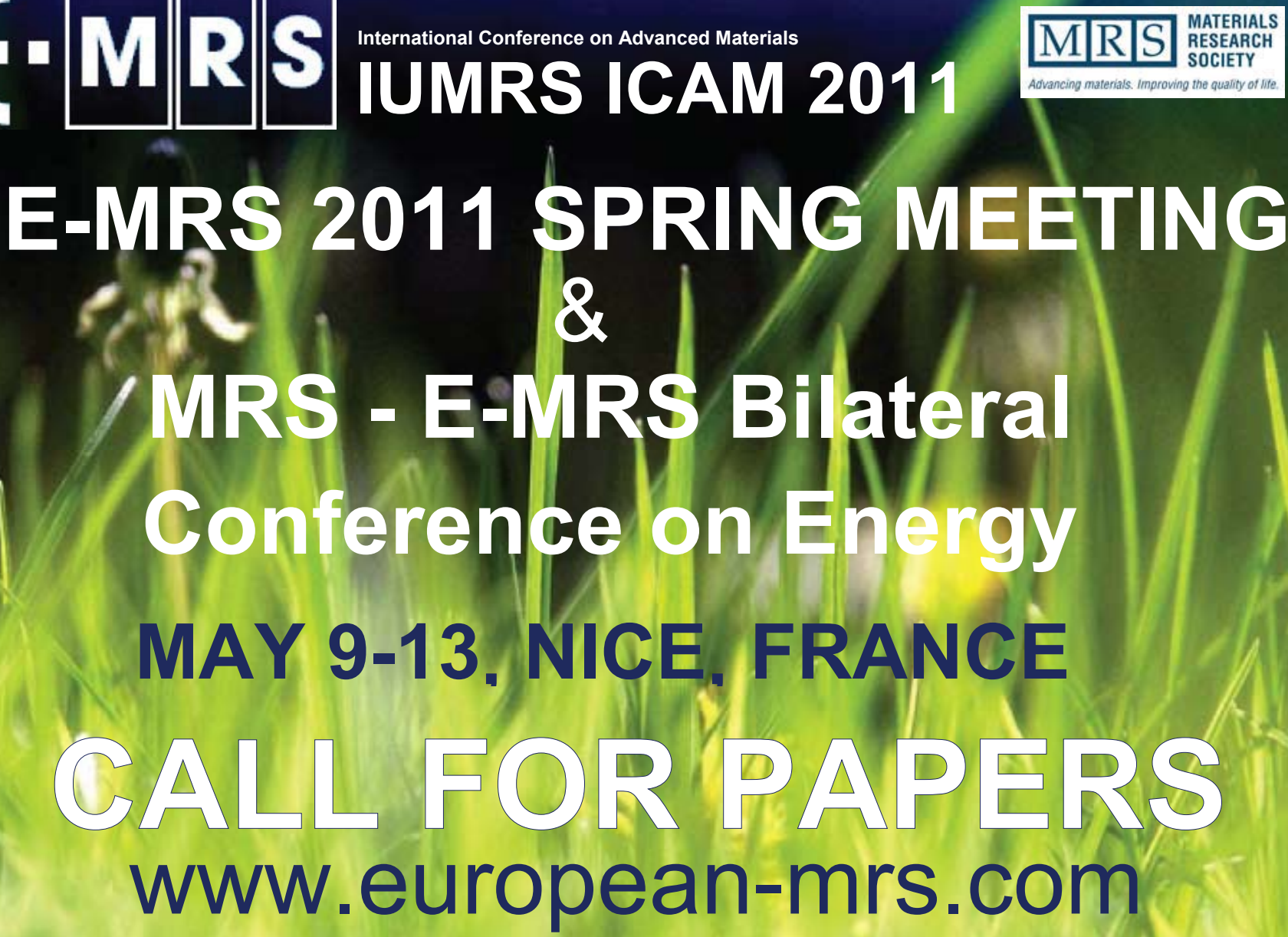

\section{ABSTRACT DEADLINE: JANUARY 14, 2011}

\section{Scheduled Symposia}

\section{THIN FILMS \& NANOMATERIALS}

A MACAN11: reconciling atomistic and continuum approaches to interfaces

B Ion beam synthesis \& modification of nanostructured materials \& surfaces

C Size-dependent properties of nanomaterials

D Synthesis, processing and characterization of nanoscale multi functional oxide films III

\section{ELECTRONICS \& PHOTONICS}

E From photophysics to optoelectronics of zero- and onedimensional nanomaterials

F Group III nitrides and their heterostructures for electronics and photonics

G Semiconductor nanostructures towards electronic and optoelectronic device applications

$H$ Indium nitride and related alloys

I Transport and photonics in Si-based nanodevices

\section{MATERIALS FABRICATION \& CHARACTERIZATIONS}

J Laser materials processing for micro and nano applications $K$ Protective coatings and thin films

L Basic research on ionic - covalent materials for nuclear applications

M X-ray techniques for materials research - from laboratory sources to free electron lasers

\section{ORGANIC \& BIOINSPIRED MATERIALS}

$\mathrm{N}$ Controlling and characterising the structure of organic semiconductor films

O Bio-nanomaterials for imaging, sensing and actuating $P$ Bio-inspired and bio-integrated materials as new frontiers Hadriorifíatérizall's $\$ 2010.725$ Published online by Cambridge University Press

\section{BILATERAL CONFERENCE ON ENERGY}

Q Engineering of wide bandgap semiconductor materials for energy saving

R Advanced inorganic materials and concepts for photovoltaics

S Organic photovoltaics: science and technology (OPV)

T Materials for solar hydrogen via photo electrochemical production

$U$ Nano energy: energy transduction at the nanoscale for energy conversion devices and energy storage systems

v Nuclear materials

W Materials Science for Large Scale Energy Storage $X$ Fuel Cells Energy Conversion

$Y$ Materials Needs for Balance of Systems in Solar Conversion Z Materials Needs for Concentrated Solar Energy Conversion ZZ Advances in thermoelectric materials \& devices for energy applications

C. $M$ M $\mathrm{R}$

BP. 20

F-67037 Strasbourg cedex 2

Phone : +33 388106372 Fax : +33 388106343

emrs@emrs-strasbourg.com

www.european-mrs.com 